\title{
Travail dans le public et le privé : une intensification parallèle
}

Public and private sectors : a parallel process of work intensification

Danièle Guillemot

\section{(2) OpenEdition}

\section{Journals}

Édition électronique

URL : http://journals.openedition.org/travailemploi/5407

DOI : 10.4000/travailemploi.5407

ISSN : 1775-416X

Éditeur

DARES - Ministère du Travail

\section{Édition imprimée}

Date de publication : 15 novembre 2011

Pagination : 23-40

ISBN : 0224-4365

ISSN : 0224-4365

\section{Référence électronique}

Danièle Guillemot, "Travail dans le public et le privé : une intensification parallèle », Travail et Emploi [En ligne], 128 | octobre-décembre 2011, mis en ligne le 12 septembre 2012, consulté le 19 avril 2019 URL : http://journals.openedition.org/travailemploi/5407; DOI : 10.4000/travailemploi.5407 


\title{
Travail dans le public et le privé : une intensification parallèle
}

\author{
Danièle Guillemot(*)
}

\begin{abstract}
Le statut a-t-il protégé les fonctionnaires de l'intensification du travail observée à la fin du siècle dernier? Quels effets a pu avoir l'importation dans l'administration publique de méthodes de gestion issues du privé? En s'appuyant sur les enquêtes Conditions de travail de la Dares, l'auteure examine l'évolution depuis le milieu des années 1980 des contraintes du travail ressenties par des salariés du public et du privé exerçant des mêmes fonctions ou métiers, comme les employés administratifs, les professionnels de la santé, de la sécurité et les enseignants. Par cette approche descriptive, l'article cherche à préciser les résultats des comparaisons globales entre les secteurs public et privé, qui peuvent masquer des différences propres à l'activité, mais aussi à généraliser des observations issues de nombreux travaux monographiques. Au sein de chacun des métiers examinés, l'augmentation de la pression sur le travail ressentie par les salariés apparaît d'une ampleur comparable, qu'ils soient agents de la fonction publique ou qu'ils travaillent dans le privé, même si on peut observer certaines petites différences.
\end{abstract}

La fonction publique est-elle (encore) une sinécure? Les images de fonctionnaires assis derrière des bureaux ouverts quelques heures par jour, peu pressés d'apposer le précieux cachet de l'administration sur des papiers dont il manque toujours une pièce, hantent depuis très longtemps les imaginaires ${ }^{(1)}$. En même temps, on reconnaît aux infirmières leur dévouement, comme parfois aux ouvriers de l'équipement qui dégagent les routes, la nuit, en plein hiver. L'image de l'instituteur de campagne "hussard noir de la République» le dispute à celle du professeur souvent absent et profitant des longues vacances scolaires. Derrière ces images anciennes mais tenaces, on trouve l'idée que la sécurité de l'emploi garantie par le statut du fonctionnaire n'inciterait guère à montrer du zèle au travail. Mais aussi que certains de ces fonctionnaires - identifiés par leur métier plutôt que le statut - sont tout dévoués au service du public.

Des travaux de recherche, en sociologie, en sciences politiques ou en économie, se sont attachés à étudier les agents des administrations publiques dans différentes dimensions de la vie sociale, politique, ou du travail, souvent en comparaison avec les salariés du privé. En France, beaucoup de ces travaux ont souligné les «grandes différences » entre les deux univers, et leur persistance au cours du temps, notamment dans les modes de vie ou les choix politiques

(*) Crest-Laboratoire de sociologie quantitative; Daniele. Guillemot@ensae.fr

(1) Une simple recherche du mot «fonctionnaire» sur Google images est instructive : on y trouve à foison des dessins de personnages dormant sur leur bureau, porte fermée aux pauvres usagers faisant la queue dehors.
(De Singly, Thélot, 1988; Rouban, 2006). D’autres ont mis en évidence le poids des statuts d'emploi sur les carrières professionnelles, la structure des salaires, les recrutements (Bessière, Pouget, 2007; LuCIFORA, Meurs, 2006; Audier, 1997), ou encore les formes d'engagement dans le travail (Françors, Vlassopoulos, 2008; Piotet, 2002).

Ces travaux ont plutôt mis l'accent sur les différences persistantes entre salariés du public et du privé. Cependant, Luc Rouban (2009) souligne que la coupure entre la fonction publique et le monde des entreprises s'est atténuée depuis les années 1980, notamment sur le plan de la représentation que les fonctionnaires ont de leur rôle social et de leur travail. Ils ne se considèrent plus comme des privilégiés, et se représentent davantage comme des professionnels. Cette évolution intervient alors qu'une série de réformes des administrations publiques est lancée dans la plupart des pays occidentaux. Souvent identifiées autour de l'appellation de «nouveau management public» (NPM), ces réformes s'appuient sur l'introduction dans le secteur public de mécanismes de marché et de méthodes de gestion issues du management privé. Ayant pour objectif l'augmentation de l'efficacité économique, elles mettent l'accent sur les résultats, leur mesure et les outils d'incitation pour y parvenir (Pollitt, Bouckaert, 2005). En France, la loi organique sur les lois de finance (LOLF) votée en 2001, construite autour des principes de clarification des programmes pour le Parlement, d'indicateurs de résultats et d'autonomie de gestion (JEANNOT, 2008a), et la loi sur la mobilité ou la Révision générale des politiques publiques (RGPP) visant à réorganiser l'administration de l'État dans le cadre 
d'une diminution des effectifs de fonctionnaires, s'inscrivent dans ces approches.

En Grande-Bretagne, l'un des premiers pays à avoir introduit les réformes du NPM dès la fin des années 1970, et où les effectifs de la fonction publique ont connu des baisses très importantes dans les années 1980 et 1990, l'intensification ressentie du travail aurait été, selon l'enquête européenne sur les conditions de travail, sensiblement plus forte dans le secteur public que dans le privé, ce qui représente une exception en Europe (GreEN, 2004 ; Green, Mcintosh, 2011)). En France, de nombreuses enquêtes de terrain ont étudié les conséquences des nouvelles modalités de gestion sur le travail des salariés des organismes sociaux comme les CAF (Dubois, 1999; Weller, 1999, 2003) et des entreprises publiques privatisées ou mises en concurrence (CARTIER, 2003; Piotet, 2002), où les transformations sont plus anciennes et plus radicales qu'au cœur même de la fonction publique. Des enquêtes sur l'activité d'agents de la fonction publique révèlent une intensification de la pression temporelle et un affaiblissement du travail collectif (CRAGUE et al., 2006). Elles soulignent également la tension entre une logique de rationalisation, de recherche de rendement et un objectif de qualité et de personnalisation du service rendu à des usagers de plus en plus précarisés (BOUSSARD, 2008; Raveyre, Ughetto, 2002) tout en notant un sentiment de remise en cause du sens du travail et de son utilité pour la collectivité (LINHART, 2009; LANGUMIER, 2005).

Soulignant le regain des travaux de recherche sur l'administration depuis une quinzaine d'années, Philippe Bezes et Odile Jorn-Lambert (2010) les classent en cinq grands ensembles, allant des recherches sur la structuration des systèmes administratifs, «sous-tendues par une opposition idéal-typique inspirée des perspectives wébériennes» (p. 136), aux recherches de type microsociologique qui s'attachent à l'analyse des activités quotidiennes des agents, dans lesquelles l'institution administrative s'efface presque complètement. Les enquêtes de terrain citées plus haut relèvent de cette dernière catégorie, où «l'administration $[\ldots]$ ne différencie plus vraiment ses pratiques de celles développées dans d'autres organisations » (op. cit., p. 139). De ces travaux, on peut tirer l'impression d'ensemble d'une augmentation de la pression au travail, et, de manière liée, d'une détérioration ressentie des conditions de travail, qui rejoint les analyses portant sur l'ensemble des salariés (Askenazy et al., 2006). Pourtant, la concurrence sur les marchés et la menace du chômage étant identifiées comme deux des principales sources de l'intensification du travail (AsKENAZY, 2005 ; GREEN, MCInTOSH, 2001), on peut penser que les agents de la fonction publique - tout au moins ceux qui sont protégés par le statut de fonctionnaire - peuvent a priori davantage résister aux pressions visant à accroître les rythmes de travail que les salariés du privé (BAudelot, Gollac, 2003). Si l'intensification du travail a été générale dans les années 1980 et 1990, a-t-elle finalement été de même ampleur dans la fonction publique? En s'appuyant sur une série d'enquêtes statistiques portant sur les conditions de travail, cet article propose, suivant une approche descriptive, de comparer l'évolution des contraintes pesant sur le travail dans la fonction publique et dans les entreprises au cours des vingt dernières années. La comparaison terme à terme des réponses d'un large échantillon représentatif de salariés exerçant une même fonction ou un même métier au sein de l'administration publique ou d'entreprises privées, permet de généraliser les observations des enquêtes monographiques, tout en précisant les comparaisons globales entre secteurs public et privé qui peuvent masquer des différences propres à l'activité.

Les enquêtes Conditions de travail de la Dares interrogent tous les sept ans, depuis 1978, l'ensemble des personnes ayant un emploi, quel que soit leur statut, sur la base d'un même questionnaire portant sur divers aspects du travail : les horaires, les rythmes, les contraintes physiques et psychologiques, l'organisation. En s'appuyant sur ces enquêtes, il est possible de comparer la perception qu'ont les travailleurs du public et du privé des contraintes auxquelles ils sont soumis dans leur travail et son évolution. Les réponses des personnes interrogées permettent de construire une quantification des contraintes du travail, pour toutes les catégories de salariés et au-delà de la diversité de leur activité, et ainsi de les comparer. Les évolutions constatées entre deux enquêtes doivent cependant être analysées en tenant compte des processus sociaux d'objectivation de ce qui est considéré, à un moment donné, comme des contraintes. Ces processus d'objectivation peuvent à certaines périodes, par exemple à la suite de mouvements sociaux, toucher particulièrement certaines catégories de salariés, qui «prennent conscience» de l'importance de leurs conditions de travail (GolLAC, 1997).

Une première partie de l'article expose les différences observées en 2005, date de la dernière enquête, entre la perception des agents de la fonction publique et celle des salariés des entreprises sur quelques indicateurs de contraintes dans le travail (2). La comparaison porte sur les employés

(2) Dans la suite, les termes de «pression» ou de «contraintes » seront utilisés pour désigner un ensemble d'indicateurs produits par les enquêtes Conditions de travail et examinés ici. Ils couvrent en 2005 des déclarations de contraintes d'horaires, de rythme et de relations avec le public et, en évolution de 1984 à 2005, essentiellement des contraintes de rythme. Michel Gollac (2005), et Damien Cartron et Michel Gollac (2006) établissent un lien entre ces contraintes et les mauvaises conditions de travail en général, même si ces deux aspects du travail ne se confondent pas (un travail davantage sous pression, plus intense, pouvant dans certains cas être plus intéressant). 
administratifs, dont l'activité est relativement proche quel que soit le secteur, puis sur des métiers plus spécifiquement développés dans le secteur public : dans la santé, l'enseignement et la sécurité. Cette comparaison des différences public-privé sur des métiers très divers et au cœur de la fonction publique reprend la démarche que Nicole Guignon et Lydie VINCK (2003) avaient développée à partir de l'enquête Conditions de travail de 1998, en l'actualisant avec l'édition de 2005 et en ne retenant que quelques-unes des dimensions alors étudiées. La deuxième partie sera consacrée à l'étude comparée des évolutions entre 1984 et 2005 de certains de ces indicateurs de contraintes sur le travail. Retrouve-t-on une même dynamique d'intensification du travail pour les salariés du public et du privé dans chacun de ces métiers? Si, malgré la tendance générale à l'intensification, les évolutions observées dans les différents métiers apparaissent en partie spécifiques, le statut public ou privé différencie peu les contraintes ressenties par les salariés exerçant une même fonction ou un même métier. Pourtant, outre les éléments les plus souvent avancés pour expliquer l'intensification du travail (la pression de la concurrence et la menace du chômage), d'autres facteurs comme les nouvelles organisations instrumentées par des outils informatiques et de gestion et la désyndicalisation paraissent aussi moins marqués dans la fonction publique que dans le privé au cours de la période en revue. La troisième partie, en s'appuyant sur divers travaux empiriques en économie et sociologie, propose des pistes, hypothétiques, d'interprétation des résultats.

\section{L'activité plutôt que le statut}

Comparant les conditions de travail des agents de l'État, des hôpitaux publics et des collectivités locales avec celles des salariés des entreprises, Michel Gollac et Serge Volkoff (2007) soulignent certaines spécificités liées aux missions de service public, comme les horaires décalés ou les relations parfois difficiles avec les usagers, alors que les contraintes de type bureaucratique-industriel, comme les rythmes de travail imposés par des délais courts, sont moins répandues que dans les entreprises. Par ailleurs, l'organisation au sein des administrations publiques contribue plutôt à protéger les agents, les horaires étant plus prévisibles et la coopération plus marquée que dans le privé. Mais ces spécificités sont particulièrement le fait de certains métiers comportant des activités typiquement de service public, qui représentent près de la moitié des agents ( $45 \%$, voir encadré), comme les enseignants, les professionnels de la santé, ou les agents de la sécurité publique. Si ces métiers sont aussi exercés dans le privé, il s'agit le plus souvent d'un privé «sous statut», en raison des obligations de service public qui le concernent également. D'autres métiers sont plus transversaux et peuvent être exercés dans le public comme le privé. C'est en particulier le cas des employés administratifs de secrétariat ou de gestion. Il est à noter que, par commodité, le «public» désigne ici les trois fonctions publiques, l'État, les hôpitaux publics et les collectivités territoriales, et le «privé» les entreprises, y compris les entreprises à capitaux publics comme la Poste ou la SNCF. Il aurait été intéressant d'étudier spécifiquement les entreprises publiques, où les transformations dans la gestion du travail ont été importantes et bien documentées par des enquêtes de terrain, pouvant préfigurer des évolutions plus récentes ou à venir dans le cœur de la fonction publique (JEANNOT, 2008b). Mais le parti pris ici de centrer une partie de l'analyse sur les employés administratifs ne permet pas, en raison de la taille de l'échantillon, d'étudier séparément ces entreprises.

\section{Employés administratifs du public et du privé, les petites différences}

On comptait en 2005 un peu moins de 600000 employés des fonctions «secrétariat, saisie, accueil» et «gestion, comptabilité» dans les trois fonctions publiques, soit $20 \%$ des agents qui ne sont ni enseignants, ni professionnels de la santé ou de la sécurité (voir encadré), et 1700000 $(10 \%)$ dans les entreprises. Leur importance numérique est donc grande, ils forment le cœur de ces employés de bureau, en grande majorité des femmes, étudiés dans l'histoire longue par Delphine GARDEY (2008), et correspondent, souvent, à l'archétype du bureaucrate aux yeux du public. Mais, dispersés dans les ministères, collectivités locales, établissements publics et hôpitaux, ils ou plutôt elles sont souvent peu visibles, notamment par rapport aux catégories professionnelles à l'identité forte qu'ils côtoient dans leurs fonctions de support ${ }^{(3)}$.

(3) Danièle LinHART montre ainsi comment les agents administratifs dans les rectorats se sentent négligés face aux enseignants (LINHART, 2006). 


\section{Encadré \\ Les salariés de l’État, des hôpitaux et des collectivités locales dans les enquêtes Conditions de travail}

Les enquêtes sur les conditions de travail de la Dares, adossées jusqu'en 2005 aux enquêtes Emploi de l'Insee, permettent de distinguer, parmi les salariés, ceux qui travaillent dans un établissement appartenant à l'une des trois fonctions publiques. Mais, en raison des modalités de chiffrement du statut des employeurs, les frontières entre l'État, les hôpitaux et les collectivités locales sont mal délimitées : c'est pourquoi cet article ne cherche pas à les distinguer.

Selon l'enquête Conditions de travail de 2005, une grosse moitié de ces agents $(55 \%)$ ne sont pas des professionnels de la santé, de l'enseignement, ni de la sécurité publique (voir tableau E1-1). Qui sont-ils? Près d'une moitié sont employés, travaillant surtout dans des fonctions administratives de secrétariat-saisie ou comptabilité-gestion qui regroupent, toutes catégories confondues, un tiers des agents hors enseignants et personnels de la santé ou de la sécurité publique; un quart, en général des employés ou ouvriers, ont des fonctions de nettoyage-gardiennage ou de maintenance-logistique (entretien des bâtiments ou des voies publics : écoles, hôpitaux, routes, etc.), 9 \% travaillent dans des fonctions sociales ou d'études (chercheurs, documentalistes, assistantes sociales, animateurs, assistantes maternelles, etc.) : ce sont alors surtout des cadres ou professions intermédiaires (voir tableau E1-2). Enfin, on en compte $30 \%$ dans une fonction «autre ", qui regroupe des métiers de cadres, tels qu'inspecteurs des impôts, magistrats, ingénieurs, informaticiens, ou d'ouvriers et employés, comme les jardiniers et les cuisiniers.

Notons que, dans les trois fonctions publiques, la proportion d'agents non titulaires (13\% en 2005) est très proche de la proportion de contrats précaires (CDD, intérimaires et stagiaires) dans les entreprises (12\%).

Tableau E1 : Les métiers de l'enseignement, de la santé et de la sécurité parmi l'ensemble des salariés de l'État, des collectivités locales et des hôpitaux

\begin{tabular}{|lr|r|r|r|r|r|}
\hline & & Enseignants & $\begin{array}{c}\text { Métiers de la } \\
\text { santé }\end{array}$ & $\begin{array}{c}\text { Métiers de la } \\
\text { sécurité* }\end{array}$ & Autres & Ensemble \\
\hline \multirow{2}{*}{ Cadres } & Effectif & 419000 & 76000 & 24000 & 460000 & 978000 \\
& $\%$ & 42,8 & 7,7 & 2,4 & 47,0 & 100,0 \\
\hline \multirow{2}{*}{ Professions intermédiaires } & Effectif & 541000 & 335000 & 69000 & 590000 & 1535000 \\
& $\%$ & 35,2 & 21,8 & 4,4 & 38,4 & 100,0 \\
\hline \multirow{2}{*}{ Employés } & Effectif & 0 & 450000 & 364000 & 1315000 & 2129000 \\
& $\%$ & 0,0 & 21,1 & 17,0 & 48,2 & 100,0 \\
\hline \multirow{2}{*}{ Ouvriers } & Effectif & 0 & 0 & 0 & 362000 & 362000 \\
& $\%$ & 0,0 & 0,0 & 0,0 & 100,0 & 100,0 \\
\hline \multirow{2}{*}{ Ensemble } & Effectif & 960000 & 861000 & 456000 & 2728000 & 5005000 \\
& $\%$ & 19,2 & 17,2 & 9,1 & 54,5 & 100,0 \\
\hline
\end{tabular}

*Policiers, militaires, pompiers, personnel pénitentiaire.

Lecture : les cadres des trois f onctions publiques, au nombre de 978000 (arrondi au millier) selon l'enquête Conditions de travail de 2005, sont pour 42,8\% (419 000) des enseignants.

Source : enquête sur les Conditions de travail 2005, Dares-Insee.

Tableau E2 : Fonctions et catégories sociales des salariés de l'État, des collectivités locales et des hôpitaux, autres que les professionnels de l'enseignement, de la santé et de la sécurité (\%)

\begin{tabular}{|c|c|c|c|c|c|c|}
\hline & $\begin{array}{c}\text { Chantiers, } \\
\text { maintenance, } \\
\text { manutention, } \\
\text { logistique }\end{array}$ & $\begin{array}{c}\text { Entretien, } \\
\text { nettoyage, } \\
\text { gardiennage }\end{array}$ & $\begin{array}{l}\text { Secrétariat- } \\
\text { saisie, } \\
\text { comptabilité- } \\
\text { gestion }\end{array}$ & $\begin{array}{l}\text { Recherche, } \\
\text { études, } \\
\text { formation, } \\
\text { soins aux } \\
\text { personnes }\end{array}$ & $\begin{array}{c}\text { Autres } \\
\text { fonctions }\end{array}$ & $\begin{array}{c}\text { Ensemble } \\
\text { Effectif }\end{array}$ \\
\hline Cadres & 2,4 & 0,4 & 23,1 & 31,0 & 43,1 & 460000 \\
\hline Professions intermédiaires & 13,8 & 1,9 & 32,2 & 12,4 & 39,7 & 590000 \\
\hline Employés & 8,5 & 24,2 & 43,7 & 1,8 & 21,8 & 1315000 \\
\hline Ouvriers & 68,7 & 10,6 & 2,0 & 1,9 & 16,8 & 362000 \\
\hline Ensemble & 454000 & 371000 & 878000 & 247000 & 779000 & 2728000 \\
\hline$\%$ & 16,6 & 13,6 & 32,2 & 9,1 & 28,6 & 100 \\
\hline
\end{tabular}

Lecture : 43,7\% des 1315000 employés des trois fonctions publiques qui ne travaillent pas dans la santé ou la sécurité déclarent des fonctions de secrétariat, saisie, comptabilité ou gestion.

Source : enquête sur les Conditions de travail 2005, Dares-Insee. 
Tableau 1 : Types d'horaires, rythmes de travail et contact avec le public de l'ensemble des salariés et employés administratifs des entreprises et de la fonction publique $(\%)$

\begin{tabular}{|c|c|c|}
\hline Les salariés déclarent... & $\begin{array}{c}\text { Employés administratifs }{ }^{1} \\
\text { Fonction publique }\end{array}$ & \begin{tabular}{|c|}
$\begin{array}{c}\text { Employés administratifs } \\
\text { Entreprises }\end{array}$ \\
\end{tabular} \\
\hline Une durée hebdomadaire médiane* du travail à temps plein & $37 \mathrm{~h}$ & $35 \mathrm{~h}$ \\
\hline Travailler en moyenne plus de $40 \mathrm{~h}$ par semaine & 5 & 3 \\
\hline Travailler parfois ou souvent le dimanche ou la nuit & 12 & 10 \\
\hline Un rythme de travail dépendant de normes ou délais journaliers & 29 & 36 \\
\hline $\begin{array}{l}\text { Un rythme de travail dépendant de la demande immédiate de clients ou du } \\
\text { public }\end{array}$ & 65 & 66 \\
\hline $\begin{array}{l}\text { Un rythme de travail dépendant de la surveillance permanente de la } \\
\text { hiérarchie }\end{array}$ & 23 & 31 \\
\hline $\begin{array}{l}\text { Cumul d'une contrainte de type industriel-bureaucratique et d'une contrainte } \\
\text { marchande** }\end{array}$ & 27 & 32 \\
\hline Être obligés de se dépêcher souvent ou toujours & 41 & 48 \\
\hline Être en contact direct avec le public & 74 & 75 \\
\hline Connaître des situations de tension avec le public & 31 & 32 \\
\hline Être en contact avec des personnes en détresse ou devoir calmer des gens & 64 & 50 \\
\hline Être souvent exposés à des agressions verbales ou parfois physiques & 15 & 12 \\
\hline
\end{tabular}

${ }^{1}$ Employés déclarant exercer les fonctions «secrétariat, saisie, accueil» ou «gestion, comptabilité ».

* Médiane du nombre moyen d'heures de travail par semaine dans l'emploi principal déclaré par les employés administratifs travaillant à temps plein (variable HHC).

** Contraintes de rythme de type industriel ou bureaucratique : normes de production horaires ou quotidiennes, cadence automatique des machines, déplacement automatique du produit, dépendance des collègues; contraintes marchandes : dépendance immédiate à la demande ou contact avec les clients, le public (Gollac, 2005).

Lecture : $12 \%$ des employés administratifs de la fonction publique déclarent travailler parfois ou souvent le dimanche ou la nuit, et la moitié de ceux qui travaillent à temps plein déclarent un nombre hebdomadaire moyen de $37 \mathrm{~h}$ ou plus.

Source : Enquête sur les Conditions de travail 2005, Dares et Insee.

La durée hebdomadaire médiane du travail des employés des fonctions administratives est plus longue dans le public que dans le privé, mais ils bénéficient de davantage de jours de réduction du temps de travail (RTT) et de congés annuels ${ }^{(4)}$. Les durées excédant quarante heures hebdomadaires et les horaires décalés sont peu fréquents dans les deux secteurs (voir tableau 1 et tableau A1 en annexe) : dans l'ensemble, le régime horaire des employés administratifs, celui des «horaires de bureau», diffère peu d'un secteur à l'autre, contredisant le stéréotype séculaire selon lequel les agents publics bénéficieraient d'un régime temporel très favorable (DeveTter, 2004). Dans les deux secteurs, plus des trois quarts des employés administratifs sont en contact avec des clients ou des usagers, et les deux tiers déclarent que le rythme de leur travail dépend de la demande de ces derniers obligeant à une réponse immédiate. Ces relations avec les usagers ou clients génèrent autant de tension dans le privé que dans le public. En revanche, dans ces fonctions transversales, certaines contraintes de rythme restent plus fréquentes dans le privé, et certaines relations avec les usagers sont plus difficiles dans le public : un rythme de travail dépendant de normes horaires ou journalières, ou d'une surveillance permanente de la hiérarchie, est déclaré par $36 \%$ et $31 \%$ des employés administratifs du privé, contre $29 \%$ et $21 \%$

(4) 9,5 jours de RTT en moyenne et un peu plus de 6 semaines de congés dans la fonction publique et 6,8 jours RTT et un peu plus de 5 semaines dans le privé selon l'enquête Emploi de 2005. de ceux du public ${ }^{(5)}$; et les deux tiers des employés administratifs du public déclarent être en contact avec des personnes en détresse ou devoir calmer les gens, contre - quand même - la moitié de ceux du privé.

Dans les dimensions examinées, comme dans d'autres non reprises ici, les différences entre employés administratifs du public et du privé paraissent plutôt limitées au regard de celles qui distinguent les métiers, au sein des administrations publiques et des entreprises.

\section{Au service du public : des métiers sous tension}

Près de la moitié (44\%) des salariés de l'État, des collectivités territoriales ou des hôpitaux exercent des métiers directement liés au service public tels qu'enseignants, infirmières ou aides-soignantes, ou encore policiers. Les contraintes de leur travail sont bien souvent spécifiques, en raison notamment de la permanence de la mission qu'ils exercent, qui les conduit à travailler la nuit et le week-end, et de leur travail en contact avec des usagers souvent en situation de difficulté (maladie, agressions, etc.). Les durées de travail varient en revanche selon les

(5) Ces différences sont confirmées lorsqu'on mesure les probabilités de réponse des employés administratifs de la fonction publique à ces questions, comparativement à leurs homologues des entreprises, à l'aide d'une régression logistique comprenant différentes variables de contrôle (voir annexe, tableau A1). Les différences observées dans les statistiques descriptives qui ne sont pas significatives sont signalées dans le texte. 
Tableau 2 : Types d'horaires, rythmes de travail et contact avec le public dans les métiers de la santé du public et du privé $(\%)$

\begin{tabular}{|l|c|c|}
\hline \multicolumn{1}{|c|}{ Les salariés déclarent... } & $\begin{array}{c}\text { Hôpitaux } \\
\text { publics }\end{array}$ & $\begin{array}{c}\text { Hôpitaux } \\
\text { privés }\end{array}$ \\
\hline Travailler en moyenne plus de 40 h par semaine* & 11 & 6 \\
Travailler parfois ou souvent le dimanche ou la nuit & 84 & 71 \\
\hline Un rythme de travail dépendant de normes ou délais journaliers & 39 & 45 \\
Un rythme de travail dépendant de la demande immédiate de clients ou du public & 69 & 66 \\
Cumul d'une contrainte de temps industriel-bureaucratique et d'une contrainte marchande** & 53 & 56 \\
Être obligés de se dépêcher souvent ou toujours & 71 & 62 \\
\hline Connaître des situations de tension avec le public & 64 & 49 \\
Être en contact avec des personnes en détresse ou devoir calmer des gens & 95 & 91 \\
Être souvent exposés à des agressions verbales ou parfois à des agressions physiques & 64 & 44 \\
\hline
\end{tabular}

* Pour une durée hebdomadaire du travail de 37 h dans les hôpitaux publics et de 35 h dans les hôpitaux privés (médiane du nombre moyen d'heures de travail par semaine dans l'emploi principal, déclarée par les professionnels de la santé hospitalière travaillant à temps plein (variable HHC)).

** Contraintes de rythme de type industriel ou bureaucratique : normes de production horaires ou quotidiennes, cadence automatique des machines, déplacement automatique du produit, dépendance des collègues; contraintes marchandes : dépendance immédiate à la demande ou contact avec les clients, le public (Gollac, 2005).

Lecture : 84 \% des professionnels de la santé travaillant dans des hôpitaux publics déclarent travailler parfois ou souvent le dimanche ou la nuit, 11 \% de ceux qui travaillent à temps plein déclarent un nombre hebdomadaire moyen supérieur à $40 \mathrm{~h}$.

Source : enquête sur les Conditions de travail 2005, Dares et Insee.

métiers, plus courtes dans l'enseignement, plus longues dans la sécurité publique. Les rythmes de travail des enseignants sont peu normés et peu contraignants, au contraire des métiers de la santé et de la sécurité publique, très nombreux à devoir toujours ou souvent se dépêcher. Ces agents déclarent être débordés, manquer de temps pour faire leur travail correctement, ne pas pouvoir s'interrompre pour une courte pause, etc., si bien que ces métiers sont fréquemment décrits comme étant sous pression (Cordier, 2009; Lelane, 2005).

Ces trois ensembles de métiers répondent à des missions de service public. Néanmoins, les métiers de la santé et de l'enseignement peuvent être aussi exercés dans des organisations privées, à but lucratif ou non. Lorsque c'est le cas, les contraintes du travail, davantage liées à l'activité qu'au statut, sont proches de celles de leurs collègues du public (GUIGNON, VINCK, 2003), les établissements privés devant répondre à des obligations de service public et le statut des personnels ayant d'ailleurs souvent une certaine proximité avec celui des fonctionnaires (NAUZE-Fichet, 2004). Cependant, dans ces fonctions de la santé et de l'éducation, les organisations privées se développent généralement sur des segments spécifiques, ce qui n'est pas sans conséquence pour le travail des salariés.
La plupart des professionnels de la santé exerçant dans les hôpitaux ${ }^{(6)}$ travaillent parfois ou souvent de nuit et le dimanche quel que soit leur statut, mais la proportion est plus élevée dans le public (84\% contre $71 \%$ dans le privé, voir tableau 2 ci-dessus), où l'on déclare également pour une plus grande part devoir se dépêcher souvent ou toujours. De même, des difficultés dans les relations avec les usagers sont plus souvent déclarées dans le public, comme d'être en contact avec la détresse ou devoir faire face à des agressions verbales et physiques. On doit en revanche plus souvent respecter des normes horaires ou journalières dans les hôpitaux privés. Ces différences qui restent significatives lorsqu'on tient compte des catégories sociales et de la région (tableau A2 en annexe) peuvent en partie résulter de la prise en charge plus systématique des urgences et de l'accueil de populations en difficulté par les hôpitaux publics, alors que les cliniques privées sont davantage spécialisées dans les activités programmées en journée et en semaine.

On observe très peu de différences significatives dans les contraintes du travail des enseignants du public et du privé, lorsqu'on tient compte notamment

(6) Sur 1,6 million de salariés exerçant des métiers dans la santé - principalement des médecins ou pharmaciens salariés, des infirmières et aides-soignantes -, près de la moitié travaillent dans le privé, lucratif ou non lucratif, et un peu plus de la moitié dans le public. Au sein de cet ensemble, les salariés des hôpitaux, dont l'activité est plus homogène, sont aux deux tiers des agents de la fonction publique hospitalière. En dehors des hôpitaux, les professionnels salariés de la santé sont opticiens, assistantes dentaires, pharmaciens salariés, ou encore infirmières, psychologues et médecins scolaires, puéricultrices et aides-puéricultrices travaillant en crèche, techniciens médicaux, ambulanciers, etc. Afin de mieux neutraliser des caractéristiques propres à l'activité plutôt qu'au statut, ce sont les contraintes du travail dans les seuls hôpitaux publics et privés qui sont examinées ici. 
Tableau 3 : Types d'horaires, rythmes de travail et contact avec le public des enseignants du public et du privé (\%)

\begin{tabular}{|c|c|c|}
\hline Les salariés déclarent... & $\begin{array}{c}\text { Enseignants } \\
\text { dans le public }\end{array}$ & $\begin{array}{l}\text { Enseignants } \\
\text { dans le privé }\end{array}$ \\
\hline Travailler en moyenne plus de $40 \mathrm{~h}$ par semaine* & 19 & 14 \\
\hline Travailler parfois ou souvent le dimanche ou la nuit & 51 & 46 \\
\hline Un rythme de travail dépendant de normes ou délais journaliers & 13 & 17 \\
\hline Un rythme de travail dépendant de la demande immédiate de clients ou du public & 38 & 42 \\
\hline Cumul d'une contrainte de temps industriel-bureaucratique et d'une contrainte marchande** & 20 & 23 \\
\hline Être obligés de se dépêcher souvent ou toujours & 39 & 34 \\
\hline Connaître des situations de tension avec le public & 55 & 54 \\
\hline Être en contact avec des personnes en détresse ou devoir calmer des gens & 91 & 85 \\
\hline Être souvent exposés à des agressions verbales ou parfois à des agressions physiques & 64 & 59 \\
\hline
\end{tabular}

* Pour une durée hebdomadaire médiane du travail par semaine de 35 h dans le public comme dans le privé (nombre médian d'heures de travail par semaine dans l'emploi principal des enseignants travaillant à temps plein (variable HHC)). Les instructions aux enquêteurs précisent que les enseignants doivent déclarer le nombre moyen d'heures de cours et le temps de travail à domicile.

** Contraintes de rythme de type industriel ou bureaucratique : normes de production horaires ou quotidiennes, cadence automatique des machines, déplacement automatique du produit, dépendance des collègues; contraintes marchandes : dépendance immédiate à la demande ou contact avec les clients, le public (Gollac, 2005).

Lecture : $51 \%$ des enseignants travaillant dans le public déclarent travailler parfois ou souvent le dimanche ou la nuit (correction de copies, préparation de cours), la moitié de ceux qui travaillent à temps plein déclarent un nombre hebdomadaire moyen de $35 \mathrm{~h}$ ou plus, et $19 \%$ supérieur à 40 h.

Source : enquête sur les Conditions de travail 2005, Dares et Insee.

de la plus grande implantation du secteur privé dans l'enseignement primaire (voir tableaux 3 ci-dessus et $\mathrm{A} 3$ en annexe). Les enseignants sont peu soumis à des contraintes de rythme, qu'ils exercent dans des établissements privés ou publics. Les cas de contact avec des situations de détresse, où il faut calmer des gens (probablement les élèves) et les agressions verbales ou physiques sont cependant plus fréquemment déclarés par les enseignants d'établissements publics, qui sont présents sur tout le territoire, notamment là où se trouvent les populations en plus grande difficulté.

Dans le cas des métiers de la sécurité, les missions et activités des agents publics, de type «régalien», n'ont pas d'équivalent dans le privé. La comparaison de leurs conditions de travail avec celles des agents de sécurité privée est cependant instructive, les organismes privés investissant des activités valorisables sur le marché (7), moins soumises aux obligations de continuité de service public ou d'accueil de tous, mais où les salariés sont davantage contraints par l'obligation de satisfaire le client (BONNET, 2008). Les agents civils de sécurité et de surveillance - les vigiles, convoyeurs de fonds, enquêteurs privés, etc. - sont ainsi surtout chargés de la sécurité des biens; les policiers, militaires, pompiers, de la sécurité des personnes. C'est dans le public que l'on cumule les plus longues heures de travail, que l'on est le plus fréquemment obligé

(7) On pourrait de la même façon élargir la comparaison des conditions de travail des enseignants à celles des salariés, voire des indépendants, exerçant principalement dans la formation continue ou donnant des cours de soutien, activités pour l'essentiel assurées sous un statut privé. Les différences de conditions de travail reflètent en grande partie la place laissée vacante par le service public de l'éducation dans le domaine de l'enseignement. de se dépêcher et que l'on fait le plus face à des situations de tension, où il faut calmer des gens et essuyer des agressions physiques ou verbales (voir tableaux 4 ci-dessous et A4 en annexe).

\section{Forte croissance des contraintes de rythme dans le public comme dans le privé}

Les spécificités dans les contraintes du travail déclarées par les agents de la fonction publique lors de l'enquête de 2005 paraissent mineures au regard de ce qui rassemble les salariés exerçant un même métier ou une même fonction. Ces «petites différences» sont-elles apparues, se sontelles accentuées ou au contraire réduites au cours des deux précédentes décennies marquées par un mouvement généralisé d'intensification du travail au sein du salariat? D'un côté, les fonctionnaires restent à l'abri de la compétition marchande et de la menace du chômage, ce qui a pu freiner cette évolution. D'un autre côté, l'introduction de mécanismes de marché et l'importation de modes de gestion du privé dans le public ont pu aussi conduire à faire porter «l'effort» des agents publics au niveau de celui des salariés du privé.

La comparaison des contraintes de travail déclarées aux quatre dernières enquêtes de la Dares (1984, 1991, 1998 et 2005) par les salariés des entreprises et ceux des trois fonctions publiques dans les métiers examinés fait apparaître une évolution dans l'ensemble très proche, suivant une tendance générale bien documentée par ailleurs : c'est dans les détails qu'il faut regarder pour trouver des évolutions non pas divergentes, mais dont le rythme est plus ou moins soutenu, écarts qu'il serait intéressant 
Tableau 4 : Types d'horaires, rythmes de travail et contact avec le public dans les métiers de la sécurité publique (policiers, militaires) et privée (vigiles) (\%)

\begin{tabular}{|l|c|c|}
\hline \multicolumn{1}{|c|}{ Les salariés déclarent... } & $\begin{array}{c}\text { Métiers de la } \\
\text { sécurité publique }\end{array}$ & $\begin{array}{c}\text { Métiers de la } \\
\text { sécurité privée }\end{array}$ \\
\hline Travailler en moyenne plus de 40 h par semaine* & 41 & 9 \\
Travailler parfois ou souvent le dimanche ou la nuit & 85 & 85 \\
\hline Un rythme de travail dépendant de normes ou délais journaliers & 26 & 19 \\
Un rythme de travail dépendant de la demande immédiate de clients ou du public & 56 & 47 \\
Cumul d'une contrainte de temps industriel-bureaucratique et d'une contrainte marchande** & 37 & 35 \\
Être obligés de se dépêcher souvent ou toujours & 44 & 26 \\
\hline Connaître des situations de tension avec le public & 50 & 30 \\
Être en contact avec des personnes en détresse ou devoir calmer des gens & 81 & 63 \\
Être souvent exposés à des agressions verbales ou parfois à des agressions physiques & 61 & 43 \\
\hline
\end{tabular}

* Pour une durée hebdomadaire médiane du travail à temps plein de 40 h dans les métiers de la sécurité publique et de 35 h dans les métiers de la sécurité privée (nombre médian d'heures de travail par semaine dans l'emploi principal des professionnels de la sécurité travaillant à temps plein (variable $\mathrm{HHC})$ ).

** Contraintes de rythme de type industriel ou bureaucratique : normes de production horaires ou quotidiennes, cadence automatique des machines, déplacement automatique du produit, dépendance des collègues; contraintes marchandes : dépendance immédiate à la demande ou contact avec les clients, le public (Gollac, 2005).

Lecture : $85 \%$ des salariés des métiers de la sécurité publique déclarent travailler parfois ou souvent le dimanche ou la nuit, la moitié de ceux qui travaillent à temps plein déclarent un nombre hebdomadaire moyen de $40 \mathrm{~h}$ ou plus, et $41 \%$ strictement supérieur à $40 \mathrm{~h}$.

Source : enquête sur les Conditions de travail 2005, Dares et Insee.

de creuser - ce que l'on ne fait pas ici - mais qui apparaissent bien petits au regard des tendances communes.

Globalement, les contraintes de rythme augmentent très fortement entre 1984 et 1991, plus lentement jusqu'en 1998, se stabilisent ou baissent légèrement par la suite (voir graphiques 1 à 4 ci-dessous et tableaux $\mathrm{A} 1$ à $\mathrm{A} 4$, modèle 3 , en annexe). En 1984, elles concernaient peu de monde parmi les employés administratifs du public comme du privé : $15 \%$ déclaraient un rythme de travail déterminé par une norme horaire ou journalière, $11 \%$ et $12 \%$ un rythme dépendant des collègues, $20 \%$ dépendant de la surveillance de leur hiérarchie (voir graphiques 1). Ils étaient un peu plus nombreux à être soumis aux demandes des clients ou du public obligeant à une réponse immédiate (28\% des employés administratifs du public, $33 \%$ dans le privé). Entre 1984 et 1991, la proportion d'employés administratifs ${ }^{(8)}$ déclarant être soumis à des normes horaires ou journalières a davantage augmenté dans le privé, puis les évolutions ont été parallèles, atteignant en 2005

(8) On ne dispose pas exactement de la même question pour isoler les fonctions administratives en 1984 et dans les enquêtes ultérieures, si bien que le contour de la catégorie qui nous intéresse, les employés administratifs, est un peu différent. Si les évolutions mesurées entre 1984 et 1991 peuvent en être légèrement déformées, la comparaison entre celles enregistrées dans les secteurs public et privé ne devrait pas être significativement affectée.
$36 \%$ dans le privé et $30 \%$ dans le public ${ }^{(9)}$. À l'inverse, la contrainte marchande (ou de service), représentée par un rythme de travail dépendant de la réponse immédiate à la demande des clients ou du public, a davantage augmenté, surtout à partir de 1991, pour les employés administratifs de la fonction publique (respectivement +33 points dans le privé et +37 points dans le public). Le cumul sur les mêmes employés de ces deux types de contraintes, industrielles-bureaucratiques et marchandes ${ }^{(10)}$, augmente considérablement dans les deux secteurs, alors qu'elles tendaient auparavant à s'exclure (Gollac, VOLKOFF, 1996). L'augmentation est un peu plus importante dans le privé, mais ce n'est pas statistiquement significatif (tableau A1 en annexe).

On retrouve également dans la santé, l'enseignement et la sécurité, la forte augmentation des contraintes de rythme au cours de la deuxième moitié des années 1980, suivie d'une progression ralentie ou d'une stabilisation, le privé et le

(9) En annexe A1, le modèle 3 présente les résultats d'une régression logistique réalisée sur le cumul des années 1984 et 2005 , incluant, outre la variable sectorielle (public vs privé) et cinq variables sociodémographiques, une indicatrice pour l'année, et un terme d'interaction entre l'année et le secteur. Dans le cas du modèle prenant comme variable endogène un rythme de travail dépendant d'une norme journalière ou horaire, le coefficient de l'année 2005 est positif et significatif à $1 \%$, et celui de la fonction publique croisée avec l'année 2005, est négatif et significatif à $10 \%$, confirmant le résultat présenté. (10) Le cumul calculé ici tient compte des variables disponibles dès 1984. Contraintes «industrielles-bureaucratiques» : le rythme de travail dépend du déplacement automatique d'un produit ou d'une pièce; de la cadence automatique d'une machine; du travail des collègues, de normes de production ou délais à respecter en une journée au plus. Contraintes «marchandes» : le rythme de travail dépend d'une demande à satisfaire immédiatement. 
public se différenciant peu dans ces évolutions. L'augmentation de la proportion de salariés de la santé hospitalière déclarant diverses contraintes horaires et de rythme est particulièrement spectaculaire. Dans les deux secteurs, la proportion de salariés qui déclarent à la fois des contraintes bureaucratiques et marchandes passe en vingt ans d'environ $10 \%$ à plus de $40 \%$ (graphiques 2 ci-dessous). L'essentiel de l'évolution est enregistré entre 1984 et 1991, en même temps que la dégradation de la plupart des indicateurs de conditions de travail, ce qui a été interprété comme étant en grande partie l'effet d'un processus d'objectivation provoqué par le mouvement social des personnels hospitaliers à la fin des années 1980 (Gollac, 1997) ${ }^{(11)}$. Ces évolutions ont concerné l'ensemble des professionnels de la santé, et on n'observe pas de différences significatives entre le public et le privé (voir tableau A2, modèle 3, en annexe). Malgré les différences importantes d'activités pratiquées dans les métiers de la sécurité privée et dans l'ensemble constitué par la police, l'armée, les pompiers et les surveillants pénitentiaires, les contraintes que chacun de ces groupes rapporte augmentent dans des proportions comparables, même si l'on observe certains écarts, qui d'ailleurs se compensent partiellement d'une période à l'autre ${ }^{(12)}$. Les enseignants du privé comme ceux du public sont de leur côté également peu nombreux à déclarer en 2005 être soumis à des rythmes contraints à la fois par la demande immédiate du public et par des normes journalières ou la demande de collègues $(17 \%-$ voir graphiques 3 ci-dessous), mais ce cumul était inexistant en 1984.

\section{Graphiques 1 : Évolution des contraintes de rythme déclarées par les employés administratifs entre 1984 et 2005}
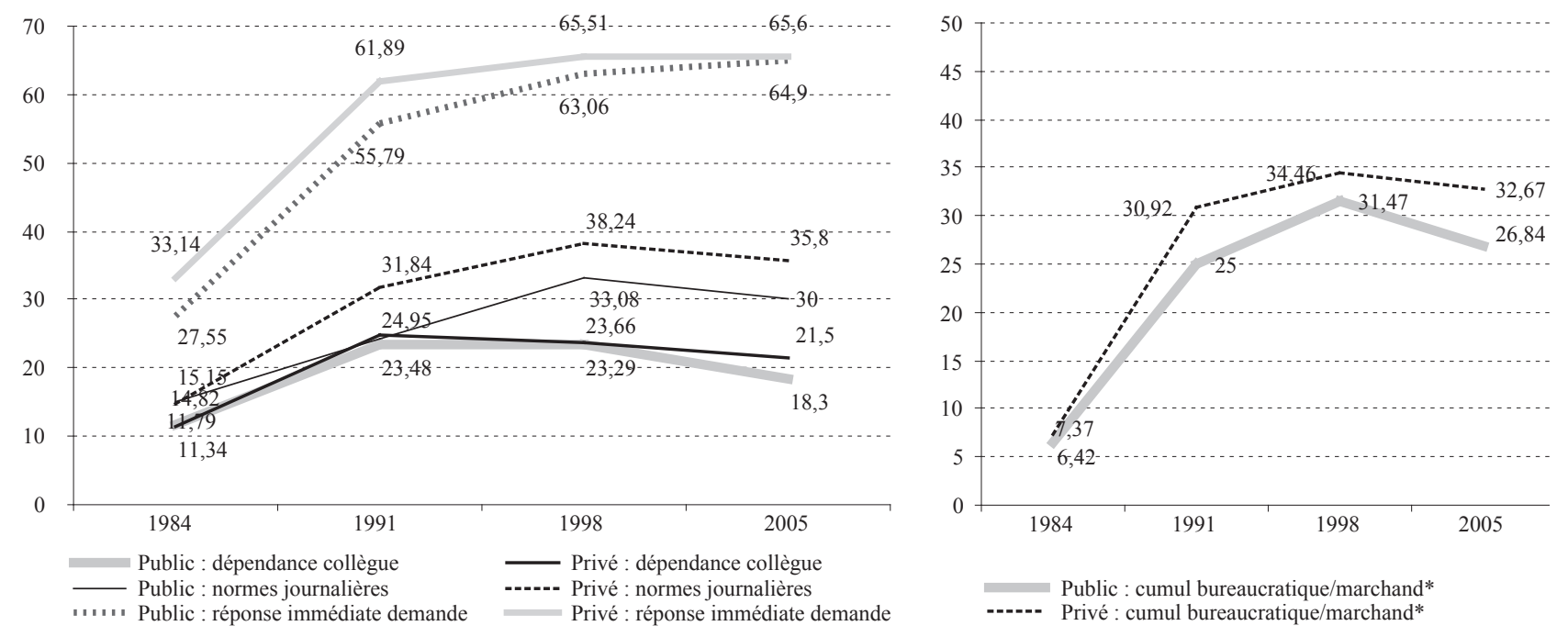

Source : Enquête sur les Conditions de travail de 1991, 1998 et 2005, Dares et Insee.

(11) Ces évolutions entre 1984 et 1991 ont surpris par leur ampleur. Elles n'ont pas seulement concerné les professionnels de la santé - on a constaté une aggravation des conditions de travail de la plupart des catégories de salariés -, mais le cas des infirmières a particulièrement attiré l'attention. GolLAC (1997) a interprété ces résultats comme étant, en partie, l'effet d'un processus d'objectivation de certaines conditions de travail qu'ont pu provoquer les conflits sociaux que cette profession a connus dans la période. Un même processus a été observé pour d'autres professions fortement mobilisées dans la période, comme les policiers (on retrouve la très forte croissance des contraintes pour les métiers de la sécurité entre 1984 et 1991) ou les cheminots.
(12) Compte tenu de la taille des échantillons (l'enquête de 2005 compte 313 enquêtés dans les métiers de la sécurité publique, et 108 dans la sécurité privée), des écarts dans les évolutions, qui peuvent paraître non négligeables, sont faiblement ou non significatifs (voir tableau A4 en annexe). 
Graphiques 2 : Évolution des contraintes de rythme déclarées dans les métiers de la santé travaillant dans les hôpitaux entre 1984 et 2005
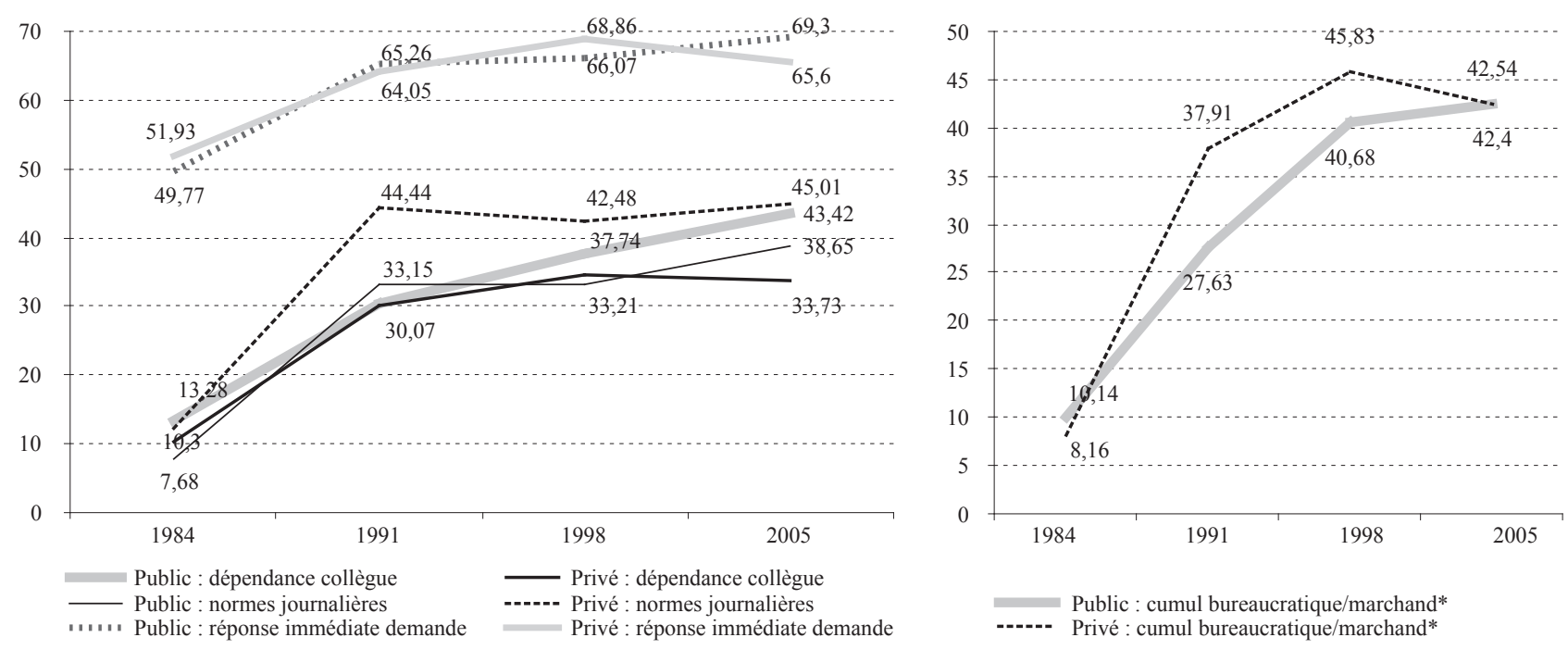

Source : Enquête Conditions de travail de 1991, 1998 et 2005, Dares et Insee.

Graphiques 3 : Évolution des contraintes de rythme déclarées par les enseignants entre 1984 et 2005
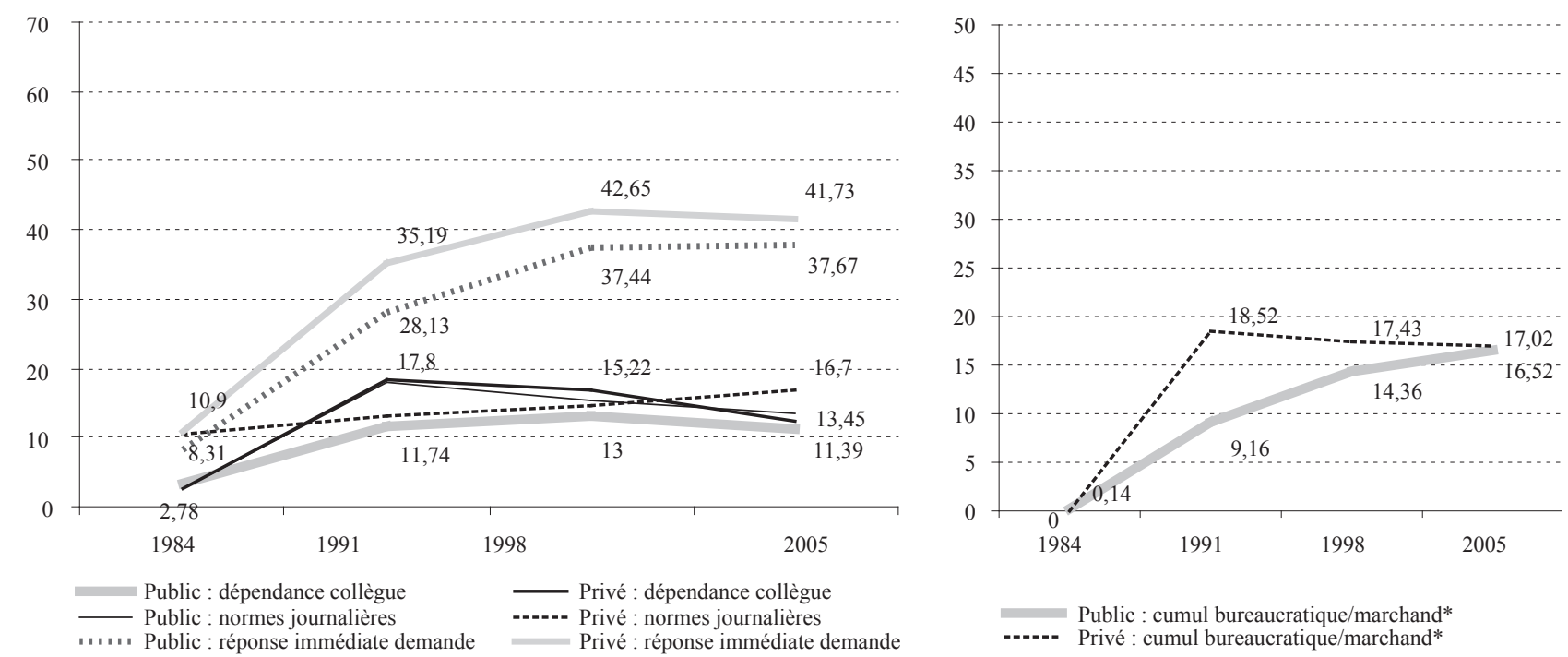

Source : Enquête Conditions de travail de 1991, 1998 et 2005, Dares et Insee.

Graphiques 4 : Évolution des contraintes de rythme déclarées dans les métiers de la sécurité entre 1984 et 2005
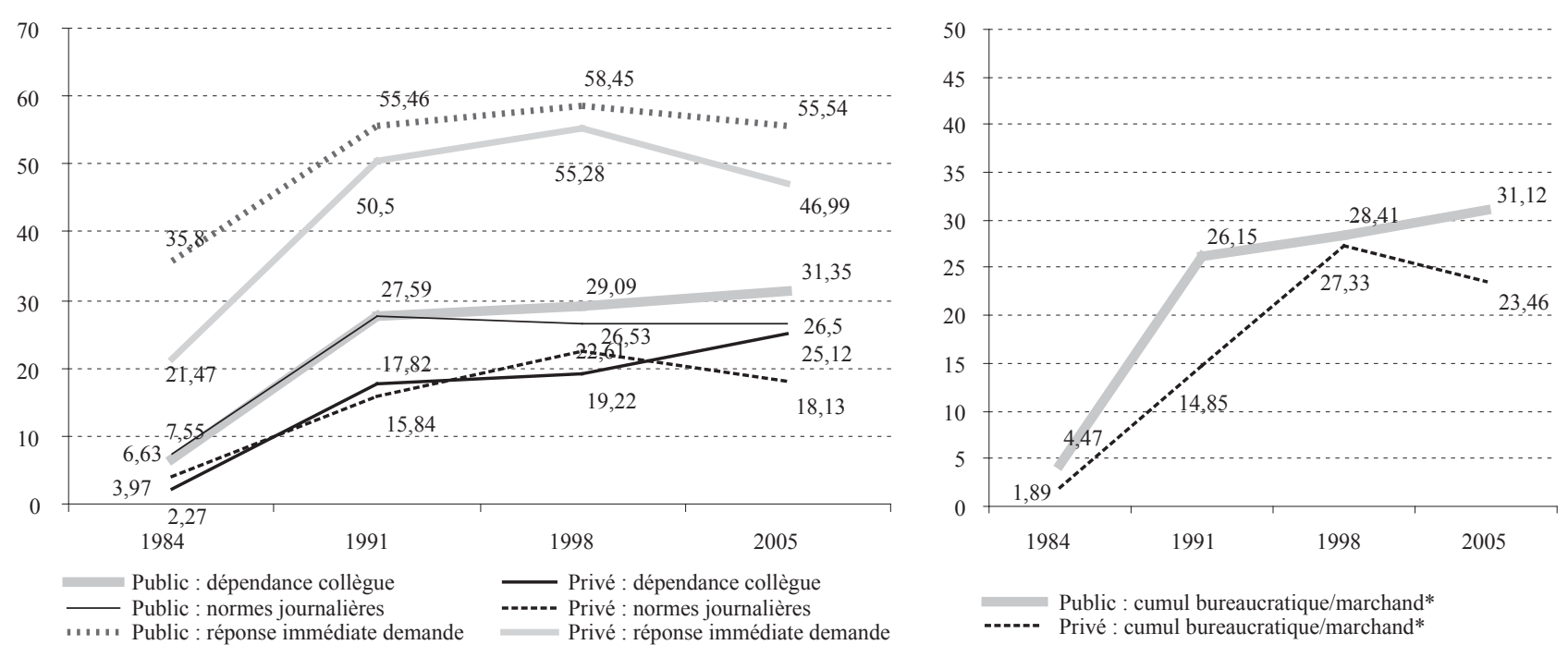

Source : Enquête Conditions de travail de 1991, 1998 et 2005, Dares et Insee. 
Les conditions de travail des salariés du public et du privé exerçant un même métier ou une même fonction étaient au début des années 1980 très proches, et elles restent, vingt ans plus tard et dans un contexte de grand changement, peu éloignées. Pour les employés administratifs, les différences les plus significatives concernent les contraintes de type bureaucratique (rythmes dépendant de normes journalières), qui ont davantage augmenté lorsqu'ils travaillent dans le privé, alors qu'à l'inverse les petits écarts de début de période constatés sur les contraintes marchandes ont disparu. Ces différences sont relativement faibles au regard de l'ampleur des évolutions sur les vingt années sous revue, démentant l'image d'une administration figée dans une bureaucratie immuable et confirmant les observations des monographies présentées en introduction.

\section{Les facteurs de l'intensification de la pression sur le travail}

De nombreux travaux réalisés au début des années 2000 sur l'ensemble du salariat, sans distinguer spécifiquement les secteurs public et privé, constataient en effet le caractère général de l'intensification du travail (AsKeNAZY et al., 2006). Francis Green et Steven McIntosh (2001) ont passé en revue les facteurs les plus fréquemment cités par les économistes pour expliquer ce phénomène : le renforcement de la concurrence dans le cadre de la mondialisation, la généralisation de l'utilisation de l'informatique au travail, la diffusion de nouvelles organisations appuyées sur des outils de gestion (juste-à-temps, normes de qualité, etc.) et celle des outils d'incitation (rémunérations à la performance). Ces transformations sont complémentaires : s'appuyant sur les innovations techniques et managériales permettant notamment un suivi en temps réel du travail (son exécution et ses résultats), les entreprises cherchent à la fois à baisser les coûts, raccourcir les délais et renforcer la variété et la qualité de leur production, pour faire face à la compétition sur le marché mondial, ces pressions étant reportées sur les salariés. Les changements d'organisation en eux-mêmes, lorsqu'ils se produisent à un rythme accéléré, peuvent d'ailleurs être sources de tension (DE CONINCK, Gollac, 2006). Les salariés sont cependant plus ou moins en mesure de résister à ces pressions pour accentuer l'intensité du travail selon le niveau de concurrence et de menace du chômage et les protections dont ils bénéficient au travers de leur statut, de l'importance des luttes sociales et de l'influence des syndicats (AsKenAZy, 2005; GreEN, 2004).

Ces facteurs de pression, d'incitation et de protection sont plus ou moins présents selon les secteurs. En particulier, la fonction publique n'est pas directement en prise avec le marché mondialisé, et le statut de fonctionnaire met à l'abri du chômage et réduit les possibilités de rémunérations individualisées ${ }^{(13)}$. La proximité de l'évolution, au cours des vingt années étudiées, des pressions sur le travail dans le public et le privé, ne paraît donc pas trouver d'explication évidente. En s'appuyant sur divers travaux empiriques en économie et sociologie, on peut tenter de discuter ici quelques pistes, hypothétiques, d'explication de ce phénomène.

\section{Un travail sous la pression du marché}

Utilisant les enquêtes européennes sur les conditions de travail menées en 1991 et 1996 dans les pays de l'UE, Green et McIntosh (2001) montrent que tous les secteurs d'activité et tous les pays étudiés sont concernés par une augmentation de l'effort au travail déclaré par les salariés ${ }^{(14)}$, mais à des degrés divers. Les salariés du secteur privé apparaissent dans la majorité des pays davantage touchés par cette intensification que dans le secteur public, résultat que les auteurs interprètent comme un proxy de l'impact des pressions du marché sur l'intensification du travail. La Grande-Bretagne fait cependant exception, les agents du public déclarant une augmentation particulièrement importante de l'intensité de leur travail, position singulière que les auteurs analysent comme le résultat de la «thatchérisation » du secteur public britannique, c'est-à-dire de l'introduction en son sein de mécanismes de marché(15).

Dans un moindre degré qu'en Grande-Bretagne, les évolutions de la sphère publique en France, comme dans les autres pays d'Europe continentale, sont aussi marquées par l'alourdissement des contraintes budgétaires, les privatisations et l'ouverture à la concurrence d'entreprises publiques, le développement de la sous-traitance au secteur privé de tâches auparavant effectuées par des agents publics et la diffusion dans les administrations publiques d'une idéologie et de pratiques gestionnaires issues du privé (Pollitt, Bouckaert, 2004;

(13) Bien sûr, les entreprises marchandes et leurs salariés ne présentent pas non plus un ensemble homogène face à la fonction publique sur ces questions : certains secteurs marchands sont davantage à l'abri de la concurrence mondiale que d'autres (construction, commerce, etc.) et les salariés de certaines grandes entreprises bénéficient souvent d'une quasi-garantie d'emploi, à commencer par ceux des anciennes grandes entreprises nationales.

(14) Les auteurs s'appuient sur deux questions, à partir desquelles ils construisent un score d'effort au travail : «How often does your main job involve working at very high speed?» et : «How often does your main job involve working to tight deadlines?» Les modalités de réponses sont proposées selon une échelle en sept positions.

(15) Il est intéressant de noter que, dans le tableau 5, p. 301, qui rassemble les résultats de modèles probit ordonnés des scores d'effort par pays, cinq d'entre eux, dont la France, se distinguent des huit autres (hors G.-B.) par l'absence de différences significatives dans l'alourdissement de l'effort rapporté par les salariés du public et du privé, ce qui apparait cohérent pour la France avec les résultats présentés ici. 
Melnik, Guillemot, 2010). Ces formes de pression indirecte du marché ont probablement contribué en France à l'intensification du travail des agents de la fonction publique, mais à l'évidence moins fortement qu'en Grande-Bretagne. Geert BoucKaERT (2003) considère ainsi que les pays d'Europe continentale ont davantage misé sur une stratégie progressive de réforme de l'État, en centrant l'évolution de leur sphère publique sur l'introduction de nouvelles méthodes de gestion plus que sur les mécanismes de marché et la mise en concurrence systématique avec le privé.

\section{Diffusion des TIC et des nouvelles pratiques de gestion}

La diffusion des TIC et des nouvelles pratiques gestionnaires, autre piste souvent évoquée pour expliquer le mouvement d'intensification du travail, a en effet été importante dans la fonction publique française. La mise en place de l'e-administration et le déploiement de progiciels de gestion intégrée (AlCAUD, LAKEL, 2004; LAU, 2004) ont notamment marqué, dans cette période, la fonction publique de l'État, si bien que la plupart de ses agents utilisent aujourd'hui un ordinateur (GUILLEMOT, Peyrin, 2010). Les enquêtes Conditions de travail confirment la généralisation de l'utilisation de l'informatique au travail : la proportion d'employés administratifs déclarant utiliser une «machine à écran» était en 1984 de $21 \%$ dans le public et $34 \%$ dans le privé. En 2005, respectivement $93 \%$ et $95 \%$ de ces employés utilisaient un outil informatique $(67 \%$ dans les métiers de la santé exercés dans le public et $59 \%$ dans le privé; $81 \%$ et $75 \%$ pour les enseignants; et $61 \%$ et $74 \%$ pour les métiers de la sécurité).

L'enquête couplée employeurs-salariés Changement organisationnel et informatisation de 2006 permet d'observer la diffusion d'outils de gestion dans les administrations de l'État, les hôpitaux et dans les entreprises marchandes (GreEnAN, GuILLemot, Kocoglu, 2010). Il ressort des premières analyses que certains outils de gestion comme les tableaux de bord se sont généralisés dans le secteur public, si bien que, même si on ne dispose pas de mesure équivalente à vingt ans de distance, il semble bien que l'on puisse parler d'une banalisation de ce qui était exceptionnel. En revanche, les outils orientés vers la qualité ou la normalisation des prestations restent plus fréquents dans le privé que dans l'administration d'État, avec cependant une diffusion très inégale selon les ministères (JEAnNot, Guillemot, 2010). Dans les hôpitaux, publics comme privés, les changements d'organisation paraissent particulièrement rapides au début des années 2000, et les outils tels que les tableaux de bord ou les mises en réseau sont maintenant très répandus (CORDIER, 2008).

Les enquêtes Conditions de travail permettent également d'observer l'utilisation de certains de ces outils de gestion au travers de ce qu'en disent les salariés. Ainsi les rémunérations liées à la performance visent directement à inciter les salariés à augmenter leur effort au travail. En 2005, $20 \%$ des salariés de l'ensemble des entreprises, et $14 \%$ des agents de la fonction publique considèrent que leur travail a un "impact important» sur leur revenu, leurs promotions ou leur carrière (et $23 \%$ un «faible impact» dans les deux secteurs). Cependant, si on s'en tient aux métiers examinés ici, la différence n'est plus significative ( $9 \%$ des employés administratifs du public et $10 \%$ dans le privé déclarent un impact important; $22 \%$ et $23 \%$ un impact faible) ou est même inversée (dans les métiers de la santé hospitalière, de la sécurité et de l'enseignement), car ce sont surtout les rémunérations ou la carrière des cadres des entreprises qui dépendent de l'évaluation du travail(16). Les enquêtés sont également interrogés, en 2005, sur des outils de prescription du travail. Les employés administratifs travaillant dans le privé sont plus nombreux à suivre une procédure de qualité et à devoir atteindre un objectif chiffré (respectivement $36 \%$ et $28 \%$, contre $25 \%$ et $23 \%$ dans le public), alors que, dans le public, ils sont davantage soumis à l'obligation d'appliquer strictement les consignes ( $43 \%$ et $38 \%$ dans le privé). Le travail des agents de la fonction publique reste largement défini par des règles, prescriptions et contrôles de type administratif voire juridique, celui des salariés du privé étant plus souvent normé par des procédures engageant leur entreprise dans leur relation contractuelle sur le marché. Enfin, l'enquête Conditions de travail de 2005 fournit un indicateur de l'intensité des changements, mais sur une période courte, en interrogeant sur les modifications de l'environnement de travail au cours de l'année passée en raison de restructurations, changements d'organisation ou dans les techniques employées. Une proportion importante des salariés, quasiment identique dans le privé et le public, déclare avoir connu de tels changements : $29 \%$ des employés administratifs du public et $28 \%$ dans le privé(17), et respectivement $37 \%$ et $33 \%$ dans les métiers de la santé, $17 \%$ et $16 \%$ des enseignants, et $35 \%$ et $27 \%$ dans les métiers de la sécurité du public et du privé (la proportion est de $26 \%$ et $27 \%$ pour l'ensemble des salariés du public et du privé). Au total, la diffusion des TIC paraît tout aussi généralisée dans les administrations publiques que dans le privé, et l'environnement organisationnel et technique du travail

(16) En 1984, $15 \%$ des salariés de l'ensemble des entreprises ( $9 \%$ des employés administratifs), et $6 \%$ des agents de la fonction publique ( $8 \%$ des employés administratifs) déclaraient alors que leur salaire ou prime dépendaient sensiblement de leur rythme de travail. La question n'étant pas libellée de la même manière qu'en 2005, on ne peut cependant pas en suivre l'évolution.

(17) Dans le détail, $13 \%$ des employés administratifs du public et $14 \%$ dans le privé déclarent un changement technique, et respectivement $18 \%$ et $19 \%$ un changement organisationnel. 
semble, dans les deux secteurs, évoluer rapidement au milieu des années 2000. Toutefois, en dépit du mouvement du nouveau management public et de grandes variations internes aux deux secteurs, les outils de gestion de la production et de la qualité restent davantage utilisés dans le privé que dans le public.

Les conséquences de ces pratiques et outils sur les rythmes de travail ne sont pas aisées à établir. Les conclusions des travaux empiriques conduisent à la prudence : GREEN (2004) soutient l'hypothèse, à partir de données britanniques, d'un changement technologique biaisé en faveur de l'augmentation de l'effort au travail, mais, pour Philippe Askenazy et Eve CAROLI (2010), si les nouvelles pratiques de gestion qu'ils étudient vont dans le sens d'une aggravation des conditions de travail, l'effet de l'utilisation des TIC est neutre ou favorable. De nombreuses enquêtes sociologiques mettent en avant la complexité des effets sur l'organisation et le travail générés par les outils de gestion, notamment ceux destinés à contrôler le travail. Les enquêtes réalisées dans le secteur public, présentées en introduction, observent plutôt un alourdissement de la pression sur les salariés lorsqu'une nouvelle organisation ou un nouvel outil est mis en place, mais Jean-Claude Moisdon (1997) et Philippe Bezes (2004) mettent en évidence des activités de détournement de la finalité de certains indicateurs de gestion et Patrick Le Gales (2004) les capacités de résistance des salariés.

\section{Le recul de la syndicalisation}

Le recul de la syndicalisation a été souvent avancé comme un facteur important d'explication de la dégradation des conditions de travail, les capacités de résistance des salariés face à la pression de leur employeur en étant affaiblies. GREEN et McIntosh (2001) ont en particulier souligné la forte corrélation, au sein de l'Union européenne, entre l'accroissement de l'indicateur d'effort fourni par les salariés entre 1991 et 1996 et le déclin de la syndicalisation. Mais en France, comme dans les autres pays, les syndicats semblent mieux résister dans le secteur public que dans le secteur privé (Amossé, Pignoni, 2006; Rouban, 2005; Booth et al., 2000) : après une forte chute, le taux de syndicalisation se maintient de la fin des années 1990 jusqu'au milieu des années 2000 à un niveau très bas dans les deux secteurs, mais particulièrement dans le privé $(5 \%$, contre $15 \%$ dans la fonction publique $\left.{ }^{(18)}\right)$. On observe également que, dans cette période, les conflits du travail sont plus nombreux dans le secteur public. Depuis la fin des années 1970, les journées d'arrêt de travail enregistrées dans les statistiques officielles - indicateur cependant très discutable de la conflictualité sociale (IRES, 2009; GiRAUD, 2006; CAMARD, 2002) ${ }^{(19)}$ - déclinent considérablement dans le secteur privé et la part de la fonction publique dans le total des jours de grève enregistrés passe de moins de $10 \%$ au début des années 1980 à près de $70 \%$ au début des années 2000, avec un pic en 1989 (Merlier, 2002). La question des conditions de travail a d'ailleurs été très présente dans les importants mouvements sociaux de la fin des années 1980 touchant particulièrement des catégories de salariés du secteur public, comme les infirmières, les agents des finances et les policiers. Les conséquences des conflits sociaux sur les réponses des salariés aux enquêtes sont cependant paradoxales, comme l'a relevé Gollac (1997, p. 25) à propos de la très forte dégradation des conditions de travail des infirmières enregistrée entre les enquêtes de 1984 et de 1991, période marquée par un conflit de grande ampleur dans les hôpitaux en 1988-1989: «[...] la dégradation des conditions de travail des infirmières [peut] être pour une part réelle (au sens faible précédemment défini : fasse l'accord des éventuels observateurs) et cette évolution [peut] être une des causes de leur mobilisation. L'ampleur des évolutions ne permet toutefois pas de douter que cette mobilisation ait eu, pour le moins, un effet amplificateur [sur la dégradation perçue des conditions de travail]».

(18) Le taux de syndicalisation est une mesure discutable du poids et de l'influence des syndicats en France. Dans les années 2000, alors que l'adhésion à un syndicat stagne à un niveau historiquement bas, la présence syndicale sur le lieu de travail augmente. En 2005, l'implantation syndicale est comparable dans le secteur public et les grandes entreprises $(70,4 \%$ des entreprises privées de plus de 100 salariés en disposent, $76,3 \%$ pour les entreprises publiques et la fonction publique) mais elle reste faible dans les petits établissements du secteur privé $(15 \%, 39 \%$ et $58 \%$ respectivement, pour les entreprises privées, publiques et administrations de moins de 100 salariés) (WolfF, 2008). Ces importantes réserves ne remettent pas en cause le constat général de recul de la puissance syndicale au cours de la période sous revue et de sa plus grande résistance dans le public.

(19) La statistique des journées individuelles non travaillées pour fait de grève (JINT), dont la collecte est par ailleurs incertaine, ne présente qu'un aspect de la conflictualité sociale, en déclin relativement à d'autres modes de mobilisation, comme les débrayages de quelques heures ou le refus d'heures supplémentaires, en hausse selon les enquêtes REPONSE de la Dares en 2002-2004 par rapport aux années 1996-1998 (CARLIER, TENRET, 2007; BÉroud et al., 2008). Ces enquêtes, restreintes au secteur marchand, ne permettent pas de quantifier ce phénomène dans la fonction publique, où on peut penser que ces formes de conflits se développent également. 


\section{Complexification du travail et baisse des coûts}

Ces différents facteurs, combinés entre eux, ont donc joué, dans tous les secteurs, dans le sens de l'augmentation générale des pressions sur le travail, mais ils ont probablement été plus modérés dans le public. Il est cependant possible, comme le suggère AskenAZy (2001), que les pressions pesant prioritairement sur les salariés du privé se soient diffusées par la culpabilisation des fonctionnaires qui côtoient dans leur entourage des salariés d'entreprises privées, culpabilisation également alimentée par la persistance du stéréotype de la fonction publique présentée comme une sinécure et par une rhétorique permanente de référence au secteur privé, pris en exemple pour l'efficacité supposée de sa gestion (RoubAn, 2009). D'autres éléments, dont attestent de nombreuses enquêtes de terrain, ont pu aussi jouer dans le sens de l'accroissement des pressions dans des activités de service, davantage présentes dans le public. La période sous revue est marquée par une attention accrue portée aux usagers devenus des clients, l'administration devant être «orientée clients » (Deroche, JEAnNot, 2004). Dans l'objectif de rapprocher l'administration de son public, des relations de service s'ajoutent au travail bureaucratique des agents administratifs, comme dans les organismes de sécurité sociale où les salariés d'exécution doivent considérer les situations individuelles des usagers là où auparavant ils appliquaient des règles uniformes (DuboIs, 1999; Weller, 1999 et 2003). Inversement, des tâches administratives s'ajoutent aux activités des professionnels les plus en contact avec le public, par exemple les soignants dans les hôpitaux qui doivent renseigner les systèmes d'information médicale (RAVEYRe, UGHetto, 2003). Ajoutant une couche supplémentaire de contraintes, les relations de service s'industrialisent à leur tour au travers de l'imposition de normes et objectifs chiffrés (Weller, 2010). Ce processus se traduit, dans ces activités, par un cumul important des contraintes bureaucratiques et marchandes. La pression peut être relayée directement par les contrôles hiérarchiques mais aussi être mise en œuvre par des outils managériaux (reporting, objectifs chiffrés, évaluation individualisée, rémunérations reliées à la performance, etc.). Cependant, on peut faire l'hypothèse que les ressorts de l'engagement altruiste dans le travail jouent aussi un rôle important (FRANÇOIS, Vlassopoulos, 2008; Linhart, 2009) : les agents du public s'efforcent de répondre aux exigences de leurs missions, lesquelles se complexifient et, en outre, entrent en conflit avec les objectifs de rendement. Les instruments de gestion importés du privé portent en effet un objectif central de réduction des coûts s'opposant à la pluralité des objectifs de la fonction publique (HECKMAN et al., 1997; JEANNOT, 2008a), et cela peut se traduire par une pression accrue sur les agents, qui s'efforcent de maintenir ces divers objectifs.

$$
\begin{array}{ll} 
& * \\
* & *
\end{array}
$$

L'approche descriptive retenue ici a permis de mettre en évidence les proximités et les «petites différences» dans l'intensité du travail de salariés travaillant dans la fonction publique ou dans une entreprise privée, et leur évolution sur vingt ans. L'intensification du travail observée dans les années 1980 et 1990 a autant touché les agents de la fonction publique que les salariés des entreprises. Malgré la protection du statut et en dépit de l'image persistante du fonctionnaire à l'abri des pressions subies par les travailleurs du privé, les contraintes de rythme ont augmenté dans des proportions comparables pour des salariés exerçant un même métier ou une même fonction dans le privé et le public, et les niveaux de contrainte constatés en 2005 au travers de l'enquête sur les Conditions de travail sont très proches : sur la question de la pression au travail, l'activité compte plus que le statut. On peut néanmoins observer certains écarts entre public et privé, notamment parmi les employés administratifs, comme une augmentation plus forte, dans le privé, des contraintes liées aux normes horaires ou journalières, et, dans la fonction publique, de la réponse immédiate à la demande. En 2005, la durée hebdomadaire du travail était un peu plus longue et les contacts avec des personnes en difficulté plus fréquents dans le public, et les contraintes de rythme plus élevées dans le privé. Passant en revue les facteurs les plus souvent mis en avant dans la littérature économique pour expliquer l'intensification du travail, on s'est interrogé sur leur pertinence dans le cas des agents de la fonction publique : si la pression du marché, la mise en concurrence, les nouveaux outils informatiques et les nouvelles pratiques managériales, la désyndicalisation, sont présents dans la fonction publique durant la période sous revue, ces phénomènes paraissent cependant y avoir été, en France, d'une moindre intensité que dans le secteur privé. Des facteurs décrits comme caractéristiques du secteur public seraient alors à prendre en considération, tels que la multiplicité des objectifs de la fonction publique et la motivation de service public portée par ses agents. Ces pistes, seulement suggérées, pourraient être creusées non seulement pour des métiers typiques du service public comme ceux de la santé, de l'enseignement ou de la sécurité, mais aussi pour les employés administratifs, dispersés dans les entreprises, ministères et établissements publics, mais qui représentent quand même 2300000 salariés, dont un quart d'agents de la fonction publique. 


\section{Bibliographie}

Amossé T., Pignoni M.-T. (2006), «La transformation du paysage syndical depuis $1945 »$, Données sociales. La société française, pp. 405-412.

Askenazy P. (2005), « Sur les sources de l'intensification », Revue économique, vol. 56, nº 2, pp. 217-236.

Askenazy P., CAroli E. (2010), «Innovative work practices, information technologies and working conditions: Evidence for France», Industrial Relations, vol. 49 (4), pp. 554-565.

Askenazy P., Cartron D., De Coninck F., Gollac M. (éds) (2006), Organisation et intensité du travail, Octarès, Toulouse.

Audier, F. (1997), «La fonction publique : un débouché majeur pour les plus diplômés », Économie et Statistique, no 304-305, pp. 137-148.

Baudelot C., Gollac M. (2003), Travailler pour être heureux? Le bonheur et le travail en France, Fayard, Paris.

Béroud S., Denis J.-M., Giraud B., Pelisse J. (2008), «Une nouvelle donne? Regain et transformations des conflits du travail» in Amossé T., Bloch-London C., Wolff L. (eds), Les relations sociales en entreprises, La Découverte, Paris.

Bessière S., Pouget J. (2007), «Les carrières dans la FPE. Premiers éléments de caractérisation», Les salaires en France, Insee, pp. 51-66.

Bezes P. (2004), «Rationalisation salariale dans l'administration française, un instrument discret», in Lascoumes P., Le Galès P., Gouverner par les instruments, Presses de Sciences Po, pp. 71-122.

Bezes P., Join-Lambert O. (2010), «Comment se font les administrations : analyser des activités administratives constituantes », Sociologie du travail, no 52, pp. 133-150.

Bonnet F. (2008), «Les effets pervers du partage de la sécurité. Polices publiques et privées dans une gare et un centre commercial», Sociologie du travail, vol. 50, no 4, pp. 447-590.

Booth A., Burda M., Calmfors L., Checchi D., Naylor R., Visser J. (2000), What Do Unions Do in Europe?, A Report for the Fondazione Rodolfo Debenedetti, Milan.

Bouckaert G. (2003), «La réforme de la gestion publique change-t-elle les systèmes administratifs?», Revue française d'administration publique, no 105-106, pp. 39-54.

Boussard V. (2008), «Plaintes de stress et modèle professionnel : quand les collectifs encadrent l'expression et la régulation du stress lié à la régulation téléphonique», in Camard S. (2002), «Comment interpréter les statistiques des grèves?», Genèses, 47, pp. 107-122.

Carlier A., Tenret E. (2007), «Des conflits du travail plus nombreux et plus diversifiés », Premières synthèses, no $08-1$, Dares.
CARTIER M. (2003), Les facteurs et leurs tournées. Un service public au quotidien, La Découverte, coll. «Textes à l'appui. Enquêtes de terrain ».

Cartron D., Gollac M. (2006), «Fastwork et maltravail», in Askenazy P., Cartron D., De Coninck F. et Gollac M., Organisation et intensité du travail, Octarès, Toulouse.

Cordier M. (2008), «Hôpitaux et cliniques en mouvement, changements d'organisation de l'offre de soins et évolution de l'attente des patients », Études et Résultats, no 633, Drees.

Crague G., De Coninck F., Delahay H., Galland J.-P. (2006), «Les transformations du travail collectif, l'impact de l'aménagement et de la réduction du temps de travail dans une administration », Sociétés contemporaines, vol. 61, no 1, pp. 119-139.

De Coninck F., Gollac M. (2006), «L'intensification du travail : de quoi parle-t-on?», in Askenazy $\mathrm{P}$ et al., Organisation et intensité du travail, Octarès, Toulouse.

Deroche L., Jeannot G. (coord.) (2004), L'Action publique au travail, Octarès, Paris.

De Singly F., Thélot C. (1989), Gens du public, gens $d u$ privé, Dunod, Paris.

Devetter F.-X. (2004), «Le temps de travail des agents publics : un régime temporel spécifique?», in L'action publique au travail, Deroche L. et Jeannot G. (dir.), Octarès, Paris.

Dubois V. (1999), La vie au guichet. Relation administrative et traitement de la misère, Economica.

François P., Vlassopoulos M. (2008), «Pro-social motivation and the delivery of social services», Cesifo Economic Studies, 54 (1), pp. 22-54.

GirAud B. (2006), «Au-delà du déclin, difficultés, rationalisation et réinvention du recours à la grève dans les stratégies confédérales des syndicats», Revue de science politique, vol. 56, no 6, pp. 943-968.

Gollac M. (1997), «Des chiffres insensés? Pourquoi et comment on donne un sens aux données statistiques», Revue française de sociologie, no 38-1, pp. 5-38.

Gollac M. (2005), «L'intensité du travail, formes et effets », Revue économique, vol. 56, n 2, pp. 195-216.

Gollac M., Volkoff S. (2007), Les conditions de travail, nouvelle édition, La Découverte, Paris.

Gollac M., Volkoff S. (1996), «Citius, altius, fortius», Actes de la recherche en sciences sociales, septembre, $\mathrm{n}^{\mathrm{o}} 114$.

GreEN F. (2004), «Why had work effort become more intense?», Industrial Relations, vol. 43, no 4, pp. 709-741.

Green F., McIntosh S. (2001), «The intensification of work in Europe », Labour Economics, vol. 8, pp. 291-308. 
Greenan N., Guillemot D. et Kocoglu Y. (dir.) (2010), "Informatisation et changements organisationnels dans les entreprises », Réseaux, vol. 28, nº 162, pp. 28-162.

Grimault S., Pernot J.-M., Ughetto P. (2005), Réforme, réactions, réactivité : trois directions du ministère de l'Économie, des Finances et de l'Industrie, rapport d'étude, Ires.

Guignon N., VInck L. (2003), «Les conditions de travail dans la fonction publique», Premières synthèses, Dares, octobre, no 40.1 .

Guillemot D., Peyrin A. (2010), «Les salariés de plus de cinquante ans et l'informatique : une comparaison public-privé», Économie et Statistique, no 437, pp. 3-28.

Heckman J., Heinrich C., Smith J. (1997), «Assessing the performance of performance standards in public bureaucracies », The American Economic Review, 87 (2), pp. 389-395.

IRES (2009), La France du travail, données, analyses, débats, Éditions de l'Atelier, Paris.

JEANNOT G. (2008a), «Réforme de la fonction publique et réorganisation de 1'État», Esprit, pp. 94-109.

JEANNOT G. (2008b), «Les fonctionnaires travaillent-ils de plus en plus? Un double inventaire des recherches sur l'activité des agents publics », Revue française de science politique, no 1 , pp. 123-140.

Jeannot G., Guillemot D. (2010), «Réformer par les outils ou réformer par les hommes? Un bilan quantitatif de la "modernisation de la gestion" de l'État», Politique et Management public, vol. 27, no 4, pp. 73-102.

LANGUMier J. (2005), «Des ouvriers de la fonction publique d'État face aux réformes de modernisation. Enquête auprès des agents d'exploitation de la DDE», Sociétés contemporaines, vol. 58, no 2, pp. 65-84.

LE GalÈs P. (2004), «Contrôle et surveillance, la restructuration de l'État en Grande-Bretagne», in Lascoumes P., Le Galès P. (2004), Gouverner par les instruments, Presses de Sciences Po, pp. 237-272.

LELANE R. (2004), «Les conditions de travail perçues par les professionnels des établissements de santé», Études et Résultats, no 335, Drees.

LinHART D. (dir.) (2006), Les différents visages de la modernisation, enquête sociologique sur les valeurs des agents de la fonction publique du Nord, La Documentation française.
Linhart D. (2009), Travailler sans les autres?, Paris, Seuil, coll. «Non conforme».

Lucifora C., Meurs D. (2006), «The public sector pay gap in France, Great Britain and Italy», Review of Income and Wealth, vol. 52, issue 1, pp. 43-59.

Melnik K., Guillemot D. (2010), «Vers une convergence du management public-privé? Une revue de littérature économique», Revue Française d'économie, n ${ }^{\circ}$ 2, pp. 167-226.

Merlier R. (2002), «Les conflits en 2000, le regain se confirme» Premières synthèses, no 09.1, Dares.

Moisdon J.-C. (1997), Du mode d'existence des outils de gestion, Paris, Seli Arslan.

NAUZE-Fichet E. (2004), «Que sait-on des différences entre public et privé?», Éducation \& Formations, n 69 , juillet.

Piotet F. (2002), Conseiller financier à La Poste. Métier, emploi, fonction ou grade? La révolution des métiers, PUF, coll. «Le Lien social».

Pollitt C., Bouckaert G. (2004), Public Management Reform. A Comparative Analysis, University Press, Oxford.

Raveyre M., UGhetto P. (2003), «Le travail, part oubliée des restructurations hospitalières », Revue française des affaires sociales, 2003/3, no 3, pp. 95-119.

Rouban L. (2005), «Public/privé: la culture sociopolitique des salariés en Europe», Cahier du Cevipof, no 40.

Rouban L. (2006), «L'univers sociopolitique des fonctionnaires français », Pouvoirs, no 117, pp. 39-54.

Rouban L. (2009), La fonction publique, La Découverte, Paris.

Weller J.-M. (1999), L'État au guichet. Sociologie cognitive du travail et modernisation administrative des services publics, Desclée de Brouwer, Paris.

Weller J.-M. (2003), «Le travail administratif, le droit et le principe de proximité», L'année sociologique, vol. 53.

Weller J.-M. (2010), «Comment les agents se soucientils des usagers?», Informations sociales, nº 158.

WolfF L. (2008), «Le paradoxe du syndicalisme français, un faible nombre d'adhérents mais des syndicats bien implantés », Dares, Premières informations et premières synthèses, no 16.1, avril. 


\section{Annexe}

Tableau A-1 : Modélisation logistique des types d'horaire, rythmes de travail et contact avec le public des employés administratifs en 2005 et 1984

\begin{tabular}{|c|c|c|c|c|c|c|c|c|c|}
\hline & $\begin{array}{c}\text { Temps plein } \\
>40 \mathrm{~h}\end{array}$ & $\begin{array}{l}\text { Travail la } \\
\text { nuit ou le } \\
\text { dimanche } \\
\text { parfois ou } \\
\text { souvent }\end{array}$ & $\begin{array}{c}\text { Ne connaît } \\
\text { pas les } \\
\text { horaires à } \\
\text { l'avance }\end{array}$ & $\begin{array}{l}\text { Rythme } \\
\text { dépend de } \\
\text { normes } \\
\text { journalières }\end{array}$ & $\mid \begin{array}{c}\text { Rythme } \\
\text { dépend de la } \\
\text { demande du } \\
\text { public }\end{array}$ & $\begin{array}{l}\text { Cumul des } \\
\text { contraintes }\end{array}$ & $\begin{array}{c}\text { Obligé de } \\
\text { se dépêcher } \\
\text { souvent }\end{array}$ & \begin{tabular}{|c|} 
Contact avec \\
la détresse \\
ou devoir \\
calmer les \\
gens
\end{tabular} & $\begin{array}{c}\text { Agressions } \\
\text { physiques } \\
\text { parfois ou } \\
\text { verbales } \\
\text { souvent }\end{array}$ \\
\hline Mod 1, 2005 & & & & & & & & & \\
\hline $\begin{array}{l}\text { Fonction publique } \\
\text { (réf : entreprises) }\end{array}$ & $\begin{array}{l}0,72 * \\
(0,39)\end{array}$ & $\begin{array}{c}0,01 \\
(0,22)\end{array}$ & $\begin{array}{l}-0,17 \\
(0,32)\end{array}$ & $\begin{array}{c}-0,44^{* *} \\
(0,15)\end{array}$ & $\begin{array}{c}0,01 \\
(0,15)\end{array}$ & $\begin{array}{l}-0,21 \\
(0,15)\end{array}$ & $\begin{array}{c}-0,46^{* * *} \\
(0,14)\end{array}$ & $\begin{array}{c}0,35 * * \\
(0,14)\end{array}$ & $\begin{array}{c}0,09 \\
(0,19)\end{array}$ \\
\hline Mod 2, 1984 & & & & & & & & & \\
\hline $\begin{array}{l}\text { Fonction publique } \\
\text { (réf : entreprises) }\end{array}$ & $\begin{array}{c}0,37 \\
(0,34)\end{array}$ & $\begin{array}{c}1,40 * * * \\
(0,19)\end{array}$ & & $\begin{array}{c}0,04 \\
(0,14)\end{array}$ & $\begin{array}{c}-0,21^{*} \\
(0,11)\end{array}$ & $\begin{array}{l}-0,04 \\
(0,19)\end{array}$ & & & \\
\hline $\begin{array}{l}\text { Mod 3, } 1984 \text { et } \\
2005\end{array}$ & & & & & & & & & \\
\hline 2005 (réf : 1984) & $\begin{array}{c}-0,47 * * \\
(0,21)\end{array}$ & $\begin{array}{c}1,36^{* * * *} \\
(0,18)\end{array}$ & & $\begin{array}{c}1,24 * * * \\
(0,10)\end{array}$ & $\begin{array}{c}1,34 * * * \\
(0,08)\end{array}$ & $\begin{array}{c}1,83 * * * \\
(0,12)\end{array}$ & & & \\
\hline $\begin{array}{l}\text { Fonction publique } \\
\text { (réf : entreprises) }\end{array}$ & $\begin{array}{c}0,25 \\
(0,30)\end{array}$ & $\begin{array}{c}1,44 * * * \\
(0,22)\end{array}$ & & $\begin{array}{c}0,09 \\
(0,16)\end{array}$ & $\begin{array}{c}0,14 \\
(0,12)\end{array}$ & $\begin{array}{c}0,03 \\
(0,21)\end{array}$ & & & \\
\hline $\begin{array}{l}2005^{*} \text { Fonction } \\
\text { publique }\end{array}$ & $\begin{array}{l}0,69^{*} \\
(0,36)\end{array}$ & $\begin{array}{c}-1,33 * * * \\
(0,25)\end{array}$ & & $\begin{array}{c}-0,30^{*} \\
(0,18)\end{array}$ & $\begin{array}{l}0,28^{*} \\
(0,15)\end{array}$ & $\begin{array}{l}-0,15 \\
(0,22)\end{array}$ & & & \\
\hline
\end{tabular}

*** : significatif au seuil de $1 \% ; * *$ : significatif au seuil de $5 \% ; *$ : significatif au seuil de $10 \%$.

Lecture : Différents modèles ont été testés. Pour les trois modèles ci-dessus, sept variables exogènes ont été retenues : statut public ou privé de l'établissement; effectif de l'établissement; âge, sexe, diplôme, statut d'emploi, précaire ou non et ZEAT (grande région) pour l'année 2005 seulement. Les coefficients sont issus de l'estimation de modèles Logit, les écarts-types sont présentés entre parenthèses.

Source : Enquête sur les Conditions de travail 2005, Dares et Insee.

Tableau A-2 : Modélisation logistique des types d'horaire, rythmes de travail et contact avec le public des professionnels de santé des hôpitaux

\begin{tabular}{|c|c|c|c|c|c|c|c|c|c|}
\hline & $\begin{array}{c}\text { Temps plein } \\
>40 \mathrm{~h}\end{array}$ & $\begin{array}{l}\text { Travail la } \\
\text { nuit ou le } \\
\text { dimanche } \\
\text { parfois ou } \\
\text { souvent }\end{array}$ & $\begin{array}{c}\text { Ne connaît } \\
\text { pas les } \\
\text { horaires à } \\
\text { l'avance }\end{array}$ & $\begin{array}{l}\text { Rythme } \\
\text { dépend de } \\
\text { normes } \\
\text { journalières }\end{array}$ & \begin{tabular}{|c|} 
Rythme \\
dépend de la \\
demande du \\
public
\end{tabular} & $\begin{array}{l}\text { Cumul des } \\
\text { contraintes }\end{array}$ & $\begin{array}{l}\text { Obligé de } \\
\text { se dépêcher } \\
\text { souvent }\end{array}$ & \begin{tabular}{|c|} 
Contact avec \\
la détresse \\
ou devoir \\
calmer les \\
gens
\end{tabular} & $\begin{array}{c}\text { Agressions } \\
\text { physiques } \\
\text { parfois ou } \\
\text { verbales } \\
\text { souvent } \\
\end{array}$ \\
\hline $\begin{array}{l}\text { Mod 1, } 2005 \\
\text { Établissements } \\
\text { publics (réf : privé) }\end{array}$ & $\begin{array}{c}0,56 \\
(0,38)\end{array}$ & $\begin{array}{c}0,83 * * * \\
(0,20)\end{array}$ & $\begin{array}{c}-0,70^{*} \\
(0,40)\end{array}$ & $\begin{array}{l}-0,07 \\
(0,16)\end{array}$ & $\begin{array}{c}0,15 \\
(0,17)\end{array}$ & $\begin{array}{c}0,04 \\
(0,16)\end{array}$ & $\begin{array}{c}0,44 * * * \\
(0,17)\end{array}$ & $\begin{array}{c}0,77 * * \\
(0,32)\end{array}$ & $\begin{array}{c}0,84 * * * \\
(0,17)\end{array}$ \\
\hline $\begin{array}{l}\text { Médecin (réf: } \\
\text { infirmier) }\end{array}$ & $\begin{array}{c}3,97 * * * \\
(0,74)\end{array}$ & $\begin{array}{c}0,06 \\
(0,56)\end{array}$ & $\begin{array}{c}1,47 \\
(0,10)\end{array}$ & $\begin{array}{c}-1,88^{* * * *} \\
(0,66)\end{array}$ & $\begin{array}{l}1,08^{*} \\
(0,58)\end{array}$ & $\begin{array}{l}-0,81 \\
(0,52)\end{array}$ & $0,15(0,53)$ & $\begin{array}{l}1,76^{* *} \\
(0,81)\end{array}$ & $\begin{array}{l}-0,81 \\
(0,52)\end{array}$ \\
\hline $\begin{array}{l}\text { Aide-soignant } \\
\text { (réf : infirmier) }\end{array}$ & $\begin{array}{c}-0,3860 \\
(0,64)\end{array}$ & $\begin{array}{c}1,60 * * * \\
(0,34)\end{array}$ & $\begin{array}{l}-1,08 \\
(0,80)\end{array}$ & $\begin{array}{c}0,38 \\
(0,29)\end{array}$ & $\begin{array}{l}-0,25 \\
(0,28)\end{array}$ & $\begin{array}{c}0,43 \\
(0,28)\end{array}$ & $\begin{array}{l}-0,07 \\
(0,28)\end{array}$ & $\begin{array}{c}1,57 * * * \\
(0,48)\end{array}$ & $\begin{array}{c}0,89 * * * \\
(0,28)\end{array}$ \\
\hline $\begin{array}{l}\text { Mod 2, } 1984 \\
\text { Établissements } \\
\text { publics (réf : privé) }\end{array}$ & $\begin{array}{c}0,25 \\
(0,28) \\
\end{array}$ & $\begin{array}{c}0,90 * * * \\
(0,22)\end{array}$ & & $\begin{array}{l}-0,10 \\
(0,21)\end{array}$ & $\begin{array}{l}-0,44 \\
(0,35) \\
\end{array}$ & $\begin{array}{c}0,18 \\
(0,36) \\
\end{array}$ & & & \\
\hline $\begin{array}{l}\text { Mod 3, } 1984 \text { et } \\
2005 \\
2005 \text { (réf : 1984) }\end{array}$ & $\begin{array}{c}-1,30 * * * \\
(0,27)\end{array}$ & $\begin{array}{c}1,08 * * * \\
(0,23)\end{array}$ & & $\begin{array}{c}1,78 * * * \\
(0,30)\end{array}$ & $\begin{array}{l}0,54 * * \\
(0,22)\end{array}$ & $\begin{array}{c}2,08 * * * \\
(0,33)\end{array}$ & & & \\
\hline $\begin{array}{l}\text { Établissements } \\
\text { publics (réf : privé) }\end{array}$ & $\begin{array}{c}0,36 \\
(0,27)\end{array}$ & $\begin{array}{c}0,99 * * * \\
(0,21)\end{array}$ & & $\begin{array}{l}-0,49 \\
(0,34)\end{array}$ & $\begin{array}{l}-0,12 \\
(0,20)\end{array}$ & $\begin{array}{c}0,28 \\
(0,34)\end{array}$ & & & \\
\hline 2005* Public & $\begin{array}{c}0,65 \\
(0,40)\end{array}$ & $\begin{array}{l}-0,27 \\
(0,28)\end{array}$ & & $\begin{array}{c}0,36 \\
(0,37)\end{array}$ & $\begin{array}{c}0,28 \\
(0,26)\end{array}$ & $\begin{array}{l}-0,22 \\
(0,38)\end{array}$ & & & \\
\hline
\end{tabular}

*** : significatif au seuil de $1 \% ; * *$ : significatif au seuil de $5 \%$; : significatif au seuil de $10 \%$.

Lecture : Différents modèles ont été testés. Pour les trois modèles ci-dessus, sept variables exogènes ont été retenues dont trois qui distinguent structurellement les professionnels de santé du public et du privé : statut public ou privé de l'établissement; profession : médecin, infirmier ou aide-soignant (la proportion de médecins est plus élevée dans les hôpitaux publics, $15 \%$ contre $7 \%$ dans le privé, et c'est l'inverse pour les infirmiers : $26 \%$ contre $38 \%$ ), et ZEAT (grande région) pour l'année 2005 (l'hôpital public est plus important dans l'Ouest avec $75 \%$ des personnels, et moins dans le Sud, 59 \%). Les modèles incluent également l'âge, le sexe, le diplôme et le statut d'emploi, précaire ou non. Les coefficients sont issus de l'estimation de modèles Logit, les écarts-types sont présentés entre parenthèses.

Source : Enquête sur les Conditions de travail 2005, Dares et Insee. 
Tableau A-3 : Modélisation logistique des types d'horaire, rythmes de travail et contact avec le public des enseignants

\begin{tabular}{|c|c|c|c|c|c|c|c|c|c|}
\hline & $\begin{array}{l}\text { Temps } \\
\text { plein }> \\
40 \mathrm{~h}\end{array}$ & $\begin{array}{l}\text { Travail la } \\
\text { nuit ou le } \\
\text { dimanche } \\
\text { parfois ou } \\
\text { souvent }\end{array}$ & $\begin{array}{c}\text { Ne connaît } \\
\text { pas les } \\
\text { horaires à } \\
\text { l'avance }\end{array}$ & $\begin{array}{l}\text { Rythme } \\
\text { dépend de } \\
\text { normes } \\
\text { journa- } \\
\text { lières }\end{array}$ & $\begin{array}{l}\text { Rythme } \\
\text { dépend } \\
\text { de la } \\
\text { demande } \\
\text { du public }\end{array}$ & $\begin{array}{l}\text { Cumul des } \\
\text { contraintes }\end{array}$ & $\begin{array}{l}\text { Obligé } \\
\text { de se } \\
\text { dépêcher } \\
\text { souvent }\end{array}$ & $\begin{array}{c}\text { Contact } \\
\text { avec la } \\
\text { détresse } \\
\text { ou devoir } \\
\text { calmer les } \\
\text { gens }\end{array}$ & $\begin{array}{c}\text { Agres- } \\
\text { sions } \\
\text { physiques } \\
\text { parfois ou } \\
\text { verbales } \\
\text { souvent }\end{array}$ \\
\hline $\begin{array}{l}\text { Mod 1, } 2005 \\
\text { Établissements } \\
\text { publics (réf : privé) }\end{array}$ & $\begin{array}{c}0,43 \\
(0,29)\end{array}$ & $\begin{array}{c}0,15 \\
(0,19)\end{array}$ & $\begin{array}{r}-0,59 \\
(0,44)\end{array}$ & $\begin{array}{l}-0,27 \\
(0,44)\end{array}$ & $\begin{array}{l}-0,08 \\
(0,19)\end{array}$ & $\begin{array}{l}-0,14 \\
(0,23)\end{array}$ & $\begin{array}{c}0,14 \\
(0,20)\end{array}$ & $\begin{array}{l}0,51^{*} \\
(0,30)\end{array}$ & $\begin{array}{c}0,46^{* *} \\
(0,23)\end{array}$ \\
\hline $\begin{array}{l}\text { Professeur } \\
\text { (réf : instituteur) }\end{array}$ & $\begin{array}{l}-0,38 \\
(0,20) \\
\end{array}$ & $\begin{array}{c}0,20 \\
(0,15)\end{array}$ & $\begin{array}{c}0,32 \\
(0,40)\end{array}$ & $\begin{array}{c}0,33 \\
(0,21)\end{array}$ & $\begin{array}{c}0,00 \\
(0,16)\end{array}$ & $\begin{array}{c}0,24 \\
(0,18)\end{array}$ & $\begin{array}{c}0,13 \\
(0,16)\end{array}$ & $\begin{array}{c}-0,70 * * * \\
(0,27) \\
\end{array}$ & $\begin{array}{c}0,44 * * \\
(0,17)\end{array}$ \\
\hline $\begin{array}{l}\text { Mod 2, } 1984 \\
\text { Établissements } \\
\text { publics (réf : privé) }\end{array}$ & & $\begin{array}{c}0,57 \\
(0,64)\end{array}$ & & $\begin{array}{c}-1,58 * * \\
(0,67)\end{array}$ & $\begin{array}{l}-0,06 \\
(0,76)\end{array}$ & & & & \\
\hline $\begin{array}{l}\text { Mod 3, } 1984 \text { et } \\
\mathbf{2 0 0 5} \\
2005 \text { (réf : 1984) }\end{array}$ & & $\begin{array}{l}1,56 * * \\
(0,66)\end{array}$ & & $\begin{array}{c}0,37 \\
(0,66)\end{array}$ & $\begin{array}{c}1,69 * * * \\
(0,64)\end{array}$ & & & & \\
\hline $\begin{array}{l}\text { Établissements } \\
\text { publics (réf : privé) }\end{array}$ & & $\begin{array}{c}0,80 \\
(0,65)\end{array}$ & & $\begin{array}{c}-1,76^{* * * *} \\
(0,67)\end{array}$ & $\begin{array}{r}-0,32 \\
(0,63)\end{array}$ & & & & \\
\hline 2005* Public & & $\begin{array}{c}0,84 \\
(0,68)\end{array}$ & & $\begin{array}{l}1,50 * * \\
(0,71)\end{array}$ & $\begin{array}{c}0,27 \\
(0,66)\end{array}$ & & & & \\
\hline
\end{tabular}

*** : significatif au seuil de $1 \% ; * *$ : significatif au seuil de $5 \% ; *$ : significatif au seuil de $10 \%$.

Lecture : Différents modèles ont été testés. Pour les trois modèles ci-dessus, sept variables exogènes ont été retenues dont trois qui distinguent structurellement les enseignants du public et du privé : statut public ou privé, professeur ou instituteur (le privé est plus implanté dans le primaire : $64 \%$ des enseignants contre $54 \%$ de ceux du public), et ZEAT (grande région) pour l'année 2005 : le privé est surtout implanté dans l'Ouest - $28 \%$ des enseignants contre $11 \%$ de ceux du public -, très peu dans le Nord et il est sous-représenté en région parisienne. Outre la grande région, qui influe significativement sur la probabilité d'horaires lourds ou décalés et de difficultés avec le public, d'autres variables géographiques ont été testées. Ainsi, les enseignants des petites communes de moins de 20000 habitants ont une probabilité significativement plus élevée de déclarer des tensions avec le public, d'être en contact avec la détresse et devoir calmer des gens, mais la spécification incluant la variable «tranches d'unité urbaines» ne change guère les résultats sur le statut public ou privé de l'établissement d'appartenance. Les modèles incluent également l'âge, le sexe, le diplôme et le statut d'emploi, précaire ou non. Les coefficients sont issus de l'estimation de modèles Logit, les écarts-types sont présentés entre parenthèses.

Source : enquête sur les Conditions de travail 2005, Dares et Insee.

Tableau A-4 : Modélisation logistique des types d'horaire, rythmes de travail et contact avec le public des professionnels de la sécurité

\begin{tabular}{|c|c|c|c|c|c|c|c|c|c|}
\hline & $\begin{array}{l}\text { Temps } \\
\text { plein }> \\
40 \mathrm{~h}\end{array}$ & $\begin{array}{l}\text { Travail la } \\
\text { nuit ou le } \\
\text { dimanche } \\
\text { parfois ou } \\
\text { souvent }\end{array}$ & $\begin{array}{c}\text { Ne connaît } \\
\text { pas les } \\
\text { horaires à } \\
\text { l'avance }\end{array}$ & $\begin{array}{l}\text { Rythme } \\
\text { dépend de } \\
\text { normes } \\
\text { journa- } \\
\text { lières }\end{array}$ & $\begin{array}{l}\text { Rythme } \\
\text { dépend } \\
\text { de la } \\
\text { demande } \\
\text { du public }\end{array}$ & $\begin{array}{l}\text { Cumul des } \\
\text { contraintes }\end{array}$ & $\begin{array}{l}\text { Obligé } \\
\text { de se } \\
\text { dépêcher } \\
\text { souvent }\end{array}$ & $\begin{array}{c}\text { Contact } \\
\text { avec la } \\
\text { détresse } \\
\text { ou devoir } \\
\text { calmer des } \\
\text { gens }\end{array}$ & $\begin{array}{c}\text { Agres- } \\
\text { sions } \\
\text { physiques } \\
\text { parfois ou } \\
\text { verbales } \\
\text { souvent }\end{array}$ \\
\hline $\begin{array}{l}\text { Mod 1, } 2005 \\
\text { Fonction publique }\end{array}$ & $\begin{array}{c}1,41 * * * \\
(0,34)\end{array}$ & $\begin{array}{c}0,02 \\
(0,33)\end{array}$ & $\begin{array}{l}-0,02 \\
(0,37)\end{array}$ & $\begin{array}{c}0,08 \\
(0,30)\end{array}$ & $\begin{array}{l}-0,09 \\
(0,24)\end{array}$ & $\begin{array}{l}-0,06 \\
(0,25)\end{array}$ & $\begin{array}{c}0,64 * * \\
(0,26)\end{array}$ & $\begin{array}{c}1,04 * * * \\
(0,28)\end{array}$ & $\begin{array}{c}0,80^{* * *} \\
(0,25)\end{array}$ \\
\hline $\begin{array}{l}\text { Mod 2, } 1984 \\
\text { Fonction publique }\end{array}$ & $\begin{array}{l}0,63 * * \\
(0,27)\end{array}$ & $\begin{array}{l}0,78 * * \\
(0,32)\end{array}$ & & $\begin{array}{c}0,46 \\
(0,58)\end{array}$ & $\begin{array}{l}0,64 * * \\
(0,28)\end{array}$ & $\begin{array}{c}0,67 \\
(0,79)\end{array}$ & & & \\
\hline $\begin{array}{l}\text { Mod 3, } 1984 \text { et } \\
\text { 2005 } \\
2005 \text { (réf : 1984) }\end{array}$ & $\begin{array}{c}-1,22 * * * \\
\quad(0,39)\end{array}$ & $\begin{array}{c}0,28 \\
(0,36)\end{array}$ & & $\begin{array}{c}1,76^{* * * *} \\
(0,57)\end{array}$ & $\begin{array}{c}1,38 * * * \\
(0,32)\end{array}$ & $\begin{array}{c}2,80^{* * *} \\
(0,75)\end{array}$ & & & \\
\hline $\begin{array}{l}\text { Fonction publique } \\
\text { (réf : entreprises) }\end{array}$ & $\begin{array}{l}0,64 * * \\
(0,26)\end{array}$ & $\begin{array}{l}0,73 * * \\
(0,31)\end{array}$ & & $\begin{array}{c}0,32 \\
(0,57)\end{array}$ & $\begin{array}{c}0,73 * * * \\
(0,28)\end{array}$ & $\begin{array}{c}0,72 \\
(0,77)\end{array}$ & & & \\
\hline $\begin{array}{l}2005 * \text { Fonction } \\
\text { publique }\end{array}$ & $\begin{array}{l}0,83 * * \\
(0,42)\end{array}$ & $\begin{array}{l}-0,62 \\
(0,44)\end{array}$ & & $\begin{array}{l}-0,17 \\
(0,63)\end{array}$ & $\begin{array}{c}-0,69 * \\
(0,36)\end{array}$ & $\begin{array}{l}-0,56 \\
(0,81)\end{array}$ & & & \\
\hline
\end{tabular}

*** : significatif au seuil de $1 \% ; * *$ : significatif au seuil de $5 \% ; *$ : significatif au seuil de $10 \%$.

Lecture : différents modèles ont été testés. Pour les trois modèles ci-dessus, sept variables exogènes ont été retenues : statut public ou privé de l'établissement; CS, âge, sexe, diplôme, statut d'emploi, précaire ou non et ZEAT (grande région) pour l'année 2005. Les coefficients sont issus de l'estimation de modèles Logit, les écarts-types sont présentés entre parenthèses.

Source : enquête sur les Conditions de travail 2005, Dares et Insee. 